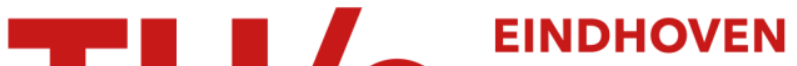 UNIVERSITY OF TECHNOLOGY
}

\section{Rigorous upscaling of the reactive flow through a pore, under dominant Peclet and Damkohler numbers}

\section{Citation for published version (APA):}

Mikelic, A., Devigne, V. M., \& Duijn, van, C. J. (2006). Rigorous upscaling of the reactive flow through a pore, under dominant Peclet and Damkohler numbers. SIAM Journal on Mathematical Analysis, 38(4), 1262-1287. https://doi.org/10.1137/050633573

DOI:

$10.1137 / 050633573$

Document status and date:

Published: 01/01/2006

\section{Document Version:}

Publisher's PDF, also known as Version of Record (includes final page, issue and volume numbers)

\section{Please check the document version of this publication:}

- A submitted manuscript is the version of the article upon submission and before peer-review. There can be important differences between the submitted version and the official published version of record. People interested in the research are advised to contact the author for the final version of the publication, or visit the $\mathrm{DOI}$ to the publisher's website.

- The final author version and the galley proof are versions of the publication after peer review.

- The final published version features the final layout of the paper including the volume, issue and page numbers.

Link to publication

\section{General rights}

Copyright and moral rights for the publications made accessible in the public portal are retained by the authors and/or other copyright owners and it is a condition of accessing publications that users recognise and abide by the legal requirements associated with these rights.

- Users may download and print one copy of any publication from the public portal for the purpose of private study or research.

- You may not further distribute the material or use it for any profit-making activity or commercial gain

- You may freely distribute the URL identifying the publication in the public portal.

If the publication is distributed under the terms of Article 25fa of the Dutch Copyright Act, indicated by the "Taverne" license above, please follow below link for the End User Agreement:

www.tue.nl/taverne

Take down policy

If you believe that this document breaches copyright please contact us at:

openaccess@tue.nl

providing details and we will investigate your claim. 


\title{
RIGOROUS UPSCALING OF THE REACTIVE FLOW THROUGH A PORE, UNDER DOMINANT PECLET AND DAMKOHLER NUMBERS*
}

\author{
ANDRO MIKELIĆ ${ }^{\dagger}$, VINCENT DEVIGNE ${ }^{\ddagger}$, AND C. J. VAN DUIJN $\S$
}

\begin{abstract}
In this paper we present a rigorous derivation of the effective model for enhanced diffusion through a narrow and long $2 \mathrm{D}$ pore. The analysis uses a singular perturbation technique. The starting point is a local pore scale model describing the transport by convection and diffusion of a reactive solute. The solute particles undergo a first-order reaction at the pore surface. The transport and reaction parameters are such that we have large, dominant Peclet and Damkohler numbers with respect to the ratio of characteristic transversal and longitudinal lengths (the small parameter $\varepsilon$ ). We give a rigorous mathematical justification of the effective behavior for small $\varepsilon$. Error estimates are presented in the energy norm as well as in $L^{\infty}$ and $L^{1}$ norms of the space variable. They guarantee the validity of the upscaled model. As a special case, we recover the well-known Taylor dispersion formula.
\end{abstract}

Key words. Taylor's dispersion, large Peclet number, singular perturbation, surface chemical reaction, large Damkohler number

AMS subject classifications. 35B25, 92E20, 76F25

DOI. $10.1137 / 050633573$

1. Introduction. We consider the transport of a reactive solute by diffusion and Poiseuille's convection in a semi-infinite 2D channel. The solute particles do not react among themselves. Instead they undergo a first-order chemical reaction at the wall of the channel. Following [12], we consider the following model for the solute concentration $c^{*}$ :

(a) transport through channel $\Omega^{*}=\left\{\left(x^{*}, y^{*}\right): 0<x^{*}<+\infty,\left|y^{*}\right|<H\right\}$ :

$$
\frac{\partial c^{*}}{\partial t^{*}}+q\left(y^{*}\right) \frac{\partial c^{*}}{\partial x^{*}}-D^{*} \frac{\partial^{2} c^{*}}{\partial\left(x^{*}\right)^{2}}-D^{*} \frac{\partial^{2} c^{*}}{\partial\left(y^{*}\right)^{2}}=0 \text { in } \Omega^{*},
$$

where $q(z)=Q^{*}\left(1-(z / H)^{2}\right)$ and where $Q^{*}$ (velocity) and $D^{*}$ (molecular diffusion) are positive constants.

(b) reaction at channel wall $\Gamma^{*}=\left\{\left(x^{*}, y^{*}\right): 0<x^{*}<+\infty,\left|y^{*}\right|=H\right\}$ :

$$
D^{*} \frac{\partial c^{*}}{\partial y^{*}}+k^{*} c^{*}=0 \quad \text { on } \Gamma^{*},
$$

where $k^{*}$ is the surface reaction coefficient.

* Received by the editors June 14, 2005; accepted for publication (in revised form) June 19, 2006; published electronically December 15, 2006.

http://www.siam.org/journals/sima/38-4/63357.html

†Institut Camille Jordan, UFR Mathématiques, Site de Gerland, Bât. A, Université Lyon 1, 50, avenue Tony Garnier, 69367 Lyon Cedex 07, France (Andro.Mikelic@univ-lyon1.fr). The research of this author was supported by the GDR MOMAS (Modélisation Mathématique et Simulations numériques liées aux problèmes de gestion des déchets nucléaires: 2439 - ANDRA, BRGM, CEA, EDF, CNRS) as a part of the project "Changements d'échelle dans la modélisation du transport multiphasique et réactif en milieux poreux: Application aux milieux fracturés et aux argiles."

${ }^{\ddagger}$ Centre SITE, Ecole Nationale Supérieure des Mines de Saint-Etienne, 158, cours Fauriel, 42023 Saint-Etienne Cedex 2, France (VincentDevigne@emse.fr).

$\S$ Department of Mathematics and Computer Science, Technische Universiteit Eindhoven, P. O. Box 513, 5600 MB Eindhoven, The Netherlands (c.j.v.duijn@tue.nl). 
The natural way of analyzing this problem is to introduce appropriate scales. This requires characteristic or reference values for the parameters and variables involved. The obvious transversal length scale is $H$. For all other quantities we use reference values denoted by the subscript $R$. Setting

$$
c=\frac{c^{*}}{\hat{c}_{R}}, x=\frac{x^{*}}{L_{R}}, y=\frac{y^{*}}{H}, t=\frac{t^{*}}{T_{R}}, Q=\frac{Q^{*}}{Q_{R}}, D=\frac{D^{*}}{D_{R}}, k=\frac{k^{*}}{k_{R}},
$$

where $L_{R}$ is the "observation distance," we obtain the dimensionless equations

$$
\frac{\partial c}{\partial t}+\frac{Q_{R} T_{R}}{L_{R}} Q\left(1-y^{2}\right) \frac{\partial c}{\partial x}-\frac{D_{R} T_{R}}{L_{R}^{2}} D \frac{\partial^{2} c}{\partial x^{2}}-\frac{D_{R} T_{R}}{H^{2}} D \frac{\partial^{2} c}{\partial y^{2}}=0 \quad \text { in } \Omega
$$

and

$$
-\frac{D D_{R}}{H k_{R}} \frac{\partial c}{\partial y}=k c \quad \text { on } \Gamma
$$

where

$$
\Omega=(0,+\infty) \times(-1,1) \text { and } \Gamma=(0,+\infty) \times\{-1,1\} .
$$

The equations involve the following time scales:

$$
\begin{aligned}
& T_{L}=\text { characteristic longitudinal time scale }=\frac{L_{R}}{Q_{R}}, \\
& T_{T}=\text { characteristic transversal time scale }=\frac{H^{2}}{D_{R}}, \\
& T_{C}=\text { superficial chemical reaction time scale }=\frac{H}{k_{R}},
\end{aligned}
$$

and the nondimensional numbers

$$
\begin{aligned}
& \left.\mathbf{P e}=\frac{L_{R} Q_{R}}{D_{R}} \quad \text { (Peclet number }\right), \\
& \mathbf{D a}=\frac{L_{R}^{2} k_{R}}{H D_{R}} \quad \text { (Damkohler number). }
\end{aligned}
$$

In this paper we fix the reference time by setting $T_{R}=T_{L}$. We are going to investigate the behavior of (4)-(5) with respect to the small parameter $\varepsilon=\frac{H}{L_{R}}$. Specifically, we will derive expressions for the effective values of the dispersion coefficient and velocity, and an effective 1D transport equation for small values of $\varepsilon$. To carry out the analysis, we need to compare the dimensionless numbers with respect to $\varepsilon$. For this purpose we set

$$
\mathbf{P e}=\varepsilon^{-\alpha} \quad \text { and } \quad \mathbf{D a}=\varepsilon^{\beta} \quad(\alpha, \beta \text { to be chosen later }) .
$$

In the absence of chemical reactions, Taylor obtained in his well-known paper [19] an explicit expression for the enhanced diffusion originating from (1). It is known as Taylor's dispersion formula. We will recover this formula as a special case in our approach by setting $\alpha=1, k=0$ and by assuming $Q=\mathcal{O}(1)$. Note that

$$
\frac{T_{T}}{T_{L}}=\frac{H Q_{R}}{D_{R}} \varepsilon=\varepsilon^{2} \mathbf{P e}=\varepsilon(\text { whenever } \alpha=1) .
$$


Introducing the dimensionless numbers in (4)-(5) and considering constant initial/ boundary conditions yields the problem

$$
\begin{gathered}
\frac{\partial c^{\varepsilon}}{\partial t}+Q\left(1-y^{2}\right) \frac{\partial c^{\varepsilon}}{\partial x}=D \varepsilon^{\alpha} \frac{\partial^{2} c^{\varepsilon}}{\partial x^{2}}+D \varepsilon^{\alpha-2} \frac{\partial^{2} c^{\varepsilon}}{\partial y^{2}} \quad \text { in } \Omega^{+} \times(0, T), \\
-D \varepsilon^{\alpha-2} \frac{\partial c^{\varepsilon}}{\partial y}=-D \frac{1}{\varepsilon^{2} \mathbf{P e}} \frac{\partial c^{\varepsilon}}{\partial y}=k \frac{\mathbf{D a}}{\mathbf{P e}} c^{\varepsilon}=k \varepsilon^{\alpha+\beta} c^{\varepsilon} \quad \text { on } \Gamma^{+} \times(0, T), \\
c^{\varepsilon}(x, y, 0)=1 \quad \text { for }(x, y) \in \Omega^{+}, \\
c^{\varepsilon}(0, y, t)=0 \text { for }(y, t) \in(0,1) \times(0, T), \\
\frac{\partial c^{\varepsilon}}{\partial y}(x, 0, t)=0 \text { for }(x, t) \in(0,+\infty) \times(0, T) .
\end{gathered}
$$

The latest condition results from the $y$-symmetry of the solution. Further

$$
\Omega^{+}=(0,+\infty) \times(0,1), \quad \Gamma^{+}=(0,+\infty) \times\{1\},
$$

and $T$ is an arbitrarily chosen positive number.

We study the behavior of this problem as $\varepsilon \searrow 0$, while keeping the coefficients $Q$, $D$, and $k$ all $\mathcal{O}(1)$. The most interesting case results when $\alpha+\beta=0$ and $0 \leq \alpha<2$, because then chemistry balances with flow in the limit as $\varepsilon \searrow 0$. Consequently, we shall restrict our attention to this situation.

In this paper we prove that the correct upscaling of (7)-(11) gives the 1D parabolic problem

$$
\left\{\begin{array}{l}
\partial_{t} c+Q\left(\frac{2}{3}+\frac{4 k}{45 D} \varepsilon^{2-\alpha}\right) \partial_{x} c+k\left(1-\frac{k}{3 D} \varepsilon^{2-\alpha}\right) c \\
=\left(D \varepsilon^{\alpha}+\frac{8}{945} \frac{Q^{2}}{D} \varepsilon^{2-\alpha}\right) \partial_{x x} c \text { in }(0,+\infty) \times(0, T), \\
\left.c\right|_{x=0}=0,\left.c\right|_{t=0}=1, \partial_{x} c \in L^{2}((0,+\infty) \times(0, T)) .
\end{array}\right.
$$

We note that for $k=0$ and $\alpha=1$ this is exactly the effective model of Taylor [19].

What is known concerning the derivation of the effective problem (EFF), with or without chemical reactions? Below we give a short overview.

- In the absence of chemical reactions, Aris [1] presented a formal derivation using the method of moments.

- For the probabilistic justification of the Taylor dispersion, in the absence of chemical reactions, we refer to the lecture notes by Caflisch and Rubinstein [4]. This approach does not give an error estimate for the approximation.

- There have been numerous attempts to give a rigorous justification for the approximation in the absence of chemical reactions. The most convincing is the near rigorous derivation using the center manifold theory by Mercer and Roberts [13]. In this paper the initial value problem is studied and the Fourier transform with respect to $x$ is applied. The center manifold theory is applied to obtain effective equations at various orders, however, without addressing the fact that one is dealing with an infinite dimensional case.

- Flow with chemistry, as described by (2), is considered by Paine, Carbonell, and Whitaker [15], who use the "single-point" closure schemes of turbulence modeling by Launder to obtain a closed model for the averaged concentration. 
These studies do not provide a rigorous mathematical derivation of the Taylor dispersion formula, and in the presence of the chemical reactions it is even not clear how to average the problem.

It should be noted that the real interest is in deriving dispersion equations for reactive flows through porous media. If we consider a porous medium comprising a bundle of capillary tubes, then we arrive at our problem. The disadvantage is that a bundle of capillary tubes represents a geometrically oversimplified model of a porous medium. Nevertheless, there is considerable insight to be gained from the analysis of our model problem.

Our technique is strongly motivated by the paper by Rubinstein and Mauri [18], where effective dispersion and convection in porous media is studied using the homogenization approach. Their analysis is based on a hierarchy of time scales. In setting up the dimensionless equations, we followed their approach. To our knowledge, the only rigorous result concerning effective dispersion in porous media in the presence of high Peclet numbers (no chemistry) and with the characteristic transport time scale is given in the recent paper by Bourgeat, Jurak, and Piatnitski [3]. Their approach uses regular solutions with compatible data for the underlying linear transport equation. They assume a high order compatibility between the initial and boundary data, involving derivatives up to order five. They construct a smooth solution of the linear transport equation, add the appropriate boundary layer and initial layer, and add the correction due to the perturbation of the mean flow. The effective solution obtained in this way is an $H^{1}$-approximation of order $\varepsilon$ and an $L^{2}$-approximation of order $\varepsilon^{2}$. However, in problems involving chemistry, one often encounters a jump between the initial value of the concentration and its value imposed at the injection boundary $x=0$. This is also the case in the experiment described by Taylor [19].

Averaging the concentration in a tube with dissolution/precipitation occurring on the wall and with $\mathbf{P e}=\mathcal{O}(1)$ is considered in [5].

For bounds on convection enhanced diffusion in porous media we refer to the work of Fannjiang, Papanicolaou, Zhikov, Kozlov, and Piatnitski. We do not give specific references because there is such an enormous number of papers on the subject. A detailed review of known results on the derivation of the effective equations of motion for the mean concentration, in the case of general heterogeneous media and transport velocities, is given in [11]. There one finds the rigorous homogenization theory for the spatio-temporal periodic velocity fields. However, in [11], the reference time is set to be the characteristic diffusion time, contrary to the choice made in [3] and the choice we have made. The case with chemical reactions, but in the absence of the transport, is considered in [2].

We note that our results also cover the case when the physical parameters result in large Peclet and small Damkohler numbers. This follows by setting $k=0$, yielding Taylor's effective equation. In fact the effective equation (20) remains valid; only the effective terms representing the surface reaction are smaller and less important.

The plan of the paper is the following. In section 2 we study the homogenized problem. It turns out that it has an explicit solution having the form of a moving Gaussian, just as the 1D boundary layers of parabolic equations, when viscosity goes to zero (see [9]). Its behavior with respect to $\varepsilon$ and $t$ is singular.

In section 3 we give a justification of a lower order approximation, using a simple energy argument. In fact this approximation does not use Taylor's dispersion formula and gives an error of the same order in $L^{\infty}\left(L^{2}\right)$ as the solution to the linear transport equation. 
In section 4 we give a formal derivation of the upscaled problem (EFF), using the approach proposed in [18].

The construction of the spatial boundary layer that takes care of the injection boundary is carried out in section 5 .

Then in sections 6 and 7 we prove that the effective concentration satisfying the corresponding 1D parabolic problem, with Taylor's diffusion coefficient and the reactive correction, is an approximation in $L^{\infty}\left(L^{2}\right)$ and in $L^{\infty}\left(L^{\infty}\right)$ to the actual physical concentration.

To satisfy the curiosity of the reader not familiar with singular perturbation techniques, we give here the simplified version of the results stated in Theorems 5-7 from section 7 . For simplicity, we compare only the physical concentration $c^{\varepsilon}$ with $c$. Keeping the correction terms is necessary to have the same precision as stated in the theorems. Throughout the paper $H(x)$ denotes Heaviside's function

$$
H(x)=1, x>0, \quad H(x)=0, x \leq 0 .
$$

Furthermore, using elementary parabolic theory one concludes that problem (7)-(11) has a unique bounded variational solution $c^{\varepsilon}$, with square integrable derivatives in $x$ and $y$. Furthermore, $c^{\varepsilon}$ belongs to $C^{\infty}$ for $x>0$ and stabilizes to 1 exponentially fast when $x \rightarrow \infty$.

THEOREM 1. Let $c$ be the unique solution of $(E F F)$ and let $\Omega_{K}=(0, K) \times(0,1)$, $K>0$. Then we have

$$
\begin{gathered}
\left\|t^{3}\left(c^{\varepsilon}-c\right)\right\|_{L^{\infty}\left(0, T ; L^{1}\left(\Omega_{K}\right)\right)} \leq C \varepsilon^{2-\alpha}, \\
\left\|t^{3}\left(c^{\varepsilon}-c\right)\right\|_{L^{\infty}\left(0, T ; L^{2}\left(\Omega_{K}\right)\right)} \leq C\left(\varepsilon^{2-5 \alpha / 4} H(1-\alpha)+\varepsilon^{3 / 2-3 \alpha / 4} H(\alpha-1)\right), \\
\left\|t^{3} \partial_{y} c^{\varepsilon}\right\|_{L^{2}\left(\Omega_{K} \times(0, T)\right)} \leq C\left(\varepsilon^{2-5 \alpha / 4} H(1-\alpha)+\varepsilon^{3 / 2-3 \alpha / 4} H(\alpha-1)\right), \\
\left\|t^{3} \partial_{x}\left(c^{\varepsilon}-c\right)\right\|_{L^{2}\left(\Omega_{K} \times(0, T)\right)} \leq C\left(\varepsilon^{2-7 \alpha / 4} H(1-\alpha)+\varepsilon^{3 / 2-5 \alpha / 4} H(\alpha-1)\right) .
\end{gathered}
$$

Note that $c$ has disappeared in estimate (15) since it is only $x$ and $t$ dependent. This estimate is superior to estimate (16) because of the large $\mathcal{O}\left(\varepsilon^{\alpha-2}\right)$ transversal diffusivity.

THEOREM 2. Let $c$ be the unique solution of (EFF). Then there exists a linear combination $\mathcal{C}_{\text {cor }}(x, y, t, \varepsilon)$ of products between polynomials in $y$ and derivatives of $c$ up to order 3 , such that

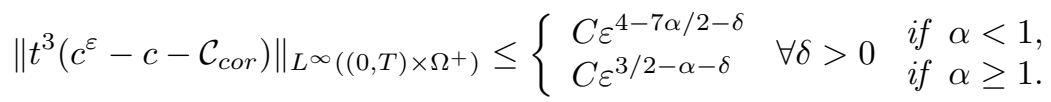

Corollary 1. Let $\alpha=1$ and $k=0$, as in Taylor's case. Then we have

$$
\begin{gathered}
\left\|t^{3}\left(c^{\varepsilon}-c-\mathcal{C}_{c o r}\right)\right\|_{L^{\infty}\left((0, T) \times \Omega^{+}\right)} \leq C \varepsilon^{1 / 2-\delta} \quad \forall \delta>0, \\
\left\|t^{3}\left(c^{\varepsilon}-c\right)\right\|_{L^{\infty}\left(0, T ; L^{1}\left(\Omega_{K}\right)\right)} \leq C \varepsilon .
\end{gathered}
$$

The expression $\mathcal{C}_{\text {cor }}(x, y, t, \varepsilon)$ is given explicitly in Theorem 7 of section 7 .

Our result could be stated in dimensional form as follows. 
Theorem 3. Let us suppose that $L_{R}>\max \left\{D_{R} / Q_{R}, Q_{R} H^{2} / D_{R}, H\right\}$. Then the upscaled dimensional approximation to (1), (2) reads

$$
\begin{aligned}
\frac{\partial c^{*, e f f}}{\partial t^{*}}+\left(\frac{2}{3}+\right. & \left.\frac{4}{45} \mathbf{D a}_{T}\right) Q^{*} \frac{\partial c^{*, e f f}}{\partial x^{*}}+\frac{k^{*}}{H}\left(1-\frac{1}{3} \mathbf{D} \mathbf{a}_{T}\right) c^{*, e f f} \\
& =D^{*}\left(1+\frac{8}{945} \mathbf{P e}_{T}^{2}\right) \frac{\partial^{2} c^{*, e f f}}{\partial\left(x^{*}\right)^{2}}
\end{aligned}
$$

where $\mathbf{P e}_{T}=\frac{Q^{*} H}{D^{*}}$ is the transversal Peclet number and $\mathbf{D a}_{T}=\frac{k^{*} H}{D^{*}}$ is the transversal Damkohler number.

We conclude this section by noting that in the known literature on boundary layers for parabolic regularization, the transport velocity is assumed to be zero at the injection boundary (see [7]). Hence our result extends the existing framework.

One could try to get even higher order approximations. Unfortunately, our procedure then leads to higher order differential operators and it is not clear if they are easy to handle. In the absence of the boundaries, higher order terms were determined in [13] using the program REDUCE.

2. Study of the upscaled diffusion-convection equation on the half-line. For $\bar{Q}, \bar{D}, \varepsilon>0$ and $\bar{k} \geq 0$, we consider the problem

$$
\left\{\begin{array}{l}
\partial_{t} u+\bar{Q} \partial_{x} u+\bar{k} u=\gamma \bar{D} \partial_{x x} u \text { in }(0,+\infty) \times(0, T), \\
\partial_{x} u \in L^{2}((0,+\infty) \times(0, T)) \\
u(x, 0)=1 \text { in }(0,+\infty), \quad u(0, t)=0 \text { at } x=0 .
\end{array}\right.
$$

The unique solution is given by the explicit formula

$$
u(x, t)=e^{-\bar{k} t}\left(1-\frac{1}{\sqrt{\pi}}\left[e^{\frac{\overline{\bar{Q} x}}{\gamma \bar{D}}} \int_{\frac{x+t \overline{\bar{Q}}}{2 \sqrt{\gamma \bar{D} t}}}^{+\infty} e^{-\eta^{2}} d \eta+\int_{\frac{x-t \bar{Q}}{2 \sqrt{\gamma \bar{D} t}}}^{+\infty} e^{-\eta^{2}} d \eta\right]\right)
$$

This expression allows us to find the exact behavior of $u$ with respect to $\gamma$. Note that for $\alpha \in[0,1]$, we will set $\gamma=\varepsilon^{\alpha}$; for $\alpha \in[1,2)$, we choose $\gamma=\varepsilon^{2-\alpha}$. The derivatives of $u$ are found using Maple, and then their norms are estimated. Since the procedure is standard, we do not give the details. In more general situations, there are no explicit solutions and these estimates could be obtained using the technique and results from [9].

By the maximum principle we have

$$
0 \leq u(x, t) \leq 1
$$

We first estimate the difference between $\chi_{\{x>\bar{Q} t\}}$ and $u$.

Lemma 1. Function $u$ satisfies the estimates

$$
\begin{gathered}
\int_{0}^{\infty}\left|e^{-\bar{k} t} \chi_{\{x>\bar{Q} t\}}-u(t, x)\right| d x=3 \sqrt{\gamma \bar{D} t} e^{-\bar{k} t}+C \gamma, \\
\left\|e^{-\bar{k} t} \chi_{\{x>\bar{Q} t\}}-u\right\|_{L^{\infty}\left(0, T ; L^{p}((0,+\infty))\right)} \leq C \gamma^{1 /(2 p)} \quad \forall p \in(1,+\infty) .
\end{gathered}
$$

For the derivatives of $u$ we have the following. 
Lemma 2. Let $\zeta$ be defined by

$$
\zeta(t)= \begin{cases}\left(\frac{t}{\bar{D} \gamma}\right)^{r} & \text { for } 0 \leq t \leq \bar{D} \gamma \\ 1 & \text { otherwise }\end{cases}
$$

with $r \geq q \geq 1$. Then

$$
\begin{gathered}
\left\|\zeta(t)\left(\left|\partial_{t} u\right|+\left|\partial_{x} u\right|\right)\right\|_{L^{q}((0, T) \times(0,+\infty))} \leq C(\gamma \bar{D})^{\min \{1 /(2 q)-1 / 2,2 / q-1\}}, q \neq 3, \\
\left\|\zeta(t)\left(\left|\partial_{t} u\right|+\left|\partial_{x} u\right|\right)\right\|_{L^{3}((0, T) \times(0,+\infty))} \leq C\left((\gamma \bar{D})^{-1} \log \left(\frac{1}{\gamma \bar{D}}\right)\right)^{1 / 3} .
\end{gathered}
$$

Next we estimate the second-order derivatives.

Lemma 3. Let $\zeta$ be defined by (26). Then

$$
\left\|\zeta(t) u_{t t}\right\|_{L^{q}((0, T) \times(0,+\infty))}+\left\|\zeta(t) u_{t x}\right\|_{L^{q}((0, T) \times(0,+\infty))}+\left\|\zeta(t) u_{x x}\right\|_{L^{q}((0, T) \times(0,+\infty))}
$$

$$
\leq C_{q}(\gamma \bar{D})^{\min \{1 /(2 q)-1,2 / q-2\}}, q \neq 3 / 2
$$

$\left\|\zeta(t) u_{t t}\right\|_{L^{3 / 2}((0, T) \times(0,+\infty))}+\left\|\zeta(t) u_{t x}\right\|_{L^{3 / 2}((0, T) \times(0,+\infty))}+\left\|\zeta(t) u_{x x}\right\|_{L^{3 / 2}((0, T) \times(0,+\infty))}$

$$
\leq C\left((\gamma \bar{D})^{-1} \log \left(\frac{1}{\gamma \bar{D}}\right)\right)^{2 / 3}
$$

For the third-order derivatives we have the following.

Lemma 4. Let $\zeta$ be defined by (26). Then

$$
\begin{gathered}
\left\|\partial_{x x x}(\zeta(t) u)\right\|_{L^{q}((0, T) \times(0,+\infty))}+\left\|\zeta(t) \partial_{x x t} u\right\|_{L^{q}((0, T) \times(0,+\infty))} \\
+\left\|\zeta(t) \partial_{x t t} u\right\|_{L^{q}((0, T) \times(0,+\infty))} \leq C_{q}(\gamma \bar{D})^{2 / q-3}, q>1 \\
\left\|\partial_{x x x}(\zeta(t) u)\right\|_{L^{1}((0, T) \times(0,+\infty))}+\left\|\zeta(t) \partial_{x x t} u\right\|_{L^{1}((0, T) \times(0,+\infty))} \\
\quad+\left\|\zeta(t) \partial_{x t t} u\right\|_{L^{1}((0, T) \times(0,+\infty))} \leq C_{1}(\gamma \bar{D})^{-1} \log \frac{1}{\gamma \bar{D}} .
\end{gathered}
$$

3. A simple $\boldsymbol{L}^{\mathbf{2}}$ error estimate. The simplest way to average problem (7)-(11) is to take the mean value with respect to $y$. Assuming that the mean of the product is the product of the means, which is in general wrong, we get the following problem for the "averaged" concentration $c_{0}^{e f f}(x, t)$ :

$$
\left\{\begin{array}{l}
\frac{\partial c_{0}^{e f f}}{\partial t}+\frac{2 Q}{3} \frac{\partial c_{0}^{e f f}}{\partial x}+k c_{0}^{e f f}=\varepsilon^{\alpha} D \frac{\partial c_{0}^{e f f}}{\partial x^{2}} \text { in }(0,+\infty) \times(0, T), \\
\partial_{x} c_{0}^{e f f} \in L^{2}((0,+\infty) \times(0, T)),\left.\quad c_{0}^{e f f}\right|_{t=0}=1,\left.\quad c_{0}^{e f f}\right|_{x=0}=0 .
\end{array}\right.
$$


This is problem (21) with $\tilde{Q}=\frac{2}{3} Q, \bar{k}=k$, and $\bar{D}=D$. The small parameter $\gamma$ is equal to $\varepsilon^{\alpha}$. For convenience we introduce the operator

$$
\mathcal{L}^{\varepsilon}(w):=\frac{\partial w}{\partial t}+Q\left(1-y^{2}\right) \frac{\partial w}{\partial x}-D \varepsilon^{\alpha}\left(\frac{\partial^{2} w}{\partial x^{2}}+\varepsilon^{-2} \frac{\partial^{2} w}{\partial y^{2}}\right) .
$$

(7)-(11) are written as

$$
\begin{aligned}
\mathcal{L}^{\varepsilon}\left(c^{\varepsilon}\right)=0 & \text { in } \quad \Omega^{+} \times(0, T), \\
c^{\varepsilon}(0, y, t)=0 \quad & \text { on } \quad(0,1) \times(0, T), \\
\partial_{y} c^{\varepsilon}(x, 0, t)=0 \quad & \text { on } \quad(0,+\infty) \times(0, T), \\
-D \varepsilon^{\alpha-2} \partial_{y} c^{\varepsilon}(x, 1, t) & =k c^{\varepsilon}(x, 1, t) \quad \text { on } \quad(0,+\infty) \times(0, T), \\
c^{\varepsilon}(x, y, 0) & =1 \quad \text { on } \quad(0,+\infty) \times(0,1) .
\end{aligned}
$$

We want to approximate $c^{\varepsilon}$ by $c_{0}^{e f f}$. For this purpose we write

$$
\begin{gathered}
\mathcal{L}^{\varepsilon}\left(c_{0}^{e f f}\right)=-k c_{0}^{e f f}+Q \partial_{x} c_{0}^{e f f}\left(1 / 3-y^{2}\right)=R^{\varepsilon}, \\
\mathcal{L}^{\varepsilon}\left(c^{\varepsilon}-c_{0}^{e f f}\right)=-R^{\varepsilon} \text { in } \Omega^{+} \times(0, T), \quad \text { and } \\
-D \varepsilon^{\alpha-2} \partial_{y}\left(c^{\varepsilon}(x, 1, t)-c_{0}^{e f f}\right)=k c^{\varepsilon}(x, 1, t) \quad \text { on }(0,+\infty) \times(0, T),
\end{gathered}
$$

and we have the following useful estimate.

Proposition 1. Let $\Psi(x)=1 /(x+1)$ and let $g^{\varepsilon}$, $\xi_{0}^{\varepsilon}$, and $R^{\varepsilon}$ be measurable functions satisfying

$$
\Psi g^{\varepsilon} \in H^{1}\left(\Omega^{+} \times(0, T)\right), \Psi \xi_{0}^{\varepsilon} \in L^{2}\left(\Omega^{+}\right), \text {and } \Psi R^{\varepsilon} \in L^{2}\left(\Omega^{+} \times(0, T)\right) .
$$

Furthermore, let $\xi \in L^{\infty}\left(\Omega^{+} \times(0, T)\right), \Psi \xi \in C\left([0, T] ; L^{2}\left(\Omega^{+}\right)\right), \Psi \nabla_{x, y} \xi \in L^{2}\left(\Omega^{+} \times\right.$ $(0, T))^{2}$ be a solution of the initial/boundary problem

$$
\begin{gathered}
\mathcal{L}^{\varepsilon}(\xi)=-R^{\varepsilon} \text { in } \Omega^{+} \times(0, T) ;\left.\quad \partial_{y} \xi\right|_{y=0}=0 \quad \text { on }(0,+\infty) \times(0, T), \\
-\left.D \varepsilon^{\alpha-2} \partial_{y} \xi\right|_{y=1}=\left.k \xi\right|_{y=1}+\left.g^{\varepsilon}\right|_{y=1} \quad \text { on }(0,+\infty) \times(0, T), \\
\left.\xi\right|_{t=0}=\xi_{0}^{\varepsilon} \quad \text { on } \Omega^{+} \quad \text { and }\left.\xi\right|_{x=0}=0 \quad \text { on }(0,1) \times(0, T) .
\end{gathered}
$$

Then we have the energy estimate

$$
\begin{gathered}
\mathcal{E}(\xi, t)=\frac{1}{2} \int_{\Omega^{+}} \Psi(x)^{2} \xi^{2}(t) d x d y+\frac{D}{2} \varepsilon^{\alpha} \int_{0}^{t} \int_{\Omega^{+}} \Psi(x)^{2} \\
\times\left\{\varepsilon^{-2}\left|\partial_{y} \xi\right|^{2}+\left|\partial_{x} \xi\right|^{2}\right\} d x d y d \tau+\left.k \int_{0}^{t} \int_{0}^{+\infty} \xi^{2}\right|_{y=1} \Psi^{2}(x) d x d \tau \\
\leq-\int_{0}^{t} \int_{\Omega^{+}} \Psi(x)^{2} R^{\varepsilon} \xi d x d y d \tau-\left.\left.\int_{0}^{t} \int_{0}^{+\infty} g^{\varepsilon}\right|_{y=1} \xi\right|_{y=1} \Psi^{2}(x) d x d \tau \\
+2 D \varepsilon^{\alpha} \int_{0}^{t} \int_{\Omega^{+}} \Psi(x)^{2} \xi^{2} d x d y d \tau+\frac{1}{2} \int_{\Omega^{+}} \Psi(x)^{2}\left(\xi_{0}^{\varepsilon}\right)^{2} d x d y .
\end{gathered}
$$

Proof. We test (43)-(45) by $\Psi^{2}(x) \xi$ to obtain

$$
\begin{gathered}
\frac{1}{2} \int_{\Omega^{+}} \xi^{2}(t) \Psi^{2}(x) d x d y+D \varepsilon^{\alpha} \int_{0}^{t} \int_{\Omega^{+}} \Psi^{2}(x)\left\{\varepsilon^{-2}\left|\partial_{y} \xi\right|^{2}+\left|\partial_{x} \xi\right|^{2}\right\} d x d y d \tau \\
+\left.k \int_{0}^{t} \int_{0}^{+\infty} \xi^{2}\right|_{y=1} \Psi^{2} d x d \tau \leq \frac{1}{2} \int_{\Omega^{+}}\left(\xi_{0}^{\varepsilon}\right)^{2} \Psi^{2}(x) d x d y \\
-\left.k \int_{0}^{t} \int_{0}^{+\infty}\left(g^{\varepsilon} \xi\right)\right|_{y=1} \Psi^{2} d x d \tau-D \varepsilon^{\alpha} \int_{0}^{t} \int_{\Omega^{+}} \partial_{x} \xi \xi \partial_{x} \Psi^{2}(x) d x d y d \tau
\end{gathered}
$$


Next we use

$$
\begin{gathered}
D \varepsilon^{\alpha} \int_{0}^{t} \int_{\Omega^{+}} \partial_{x} \xi \xi \partial_{x} \Psi^{2}(x) d x d \tau \leq \frac{D}{2} \varepsilon^{\alpha} \int_{0}^{t} \int_{\Omega^{+}} \Psi^{2}(x)\left|\partial_{x} \xi\right|^{2} d x d y d \tau \\
+2 D \varepsilon^{\alpha} \int_{0}^{t} \int_{\Omega^{+}} \Psi^{2}(x)|\xi|^{2} d x d y d \tau
\end{gathered}
$$

giving directly (46).

This simple result allows us to prove the following.

Proposition 2. There exist positive constants $C_{i}^{F}, i=1,2,3$, such that

$$
\begin{gathered}
\left\|\Psi(x)\left(c^{\varepsilon}-c_{0}^{e f f}\right)\right\|_{L^{\infty}\left(0, T ; L^{2}\left(\Omega^{+}\right)\right)} \leq \varepsilon^{1-\alpha / 2} \frac{F^{0}}{\sqrt{D}}, \\
\left\|\Psi(x) \partial_{x}\left(c^{\varepsilon}-c_{0}^{e f f}\right)\right\|_{L^{2}\left(0, T ; L^{2}\left(\Omega^{+}\right)\right)} \leq \varepsilon^{1-\alpha} \frac{F^{0}}{D}, \\
\left\|\Psi(x) \partial_{y}\left(c^{\varepsilon}-c_{0}^{e f f}\right)\right\|_{L^{2}\left(0, T ; L^{2}\left(\Omega^{+}\right)\right)} \leq \varepsilon^{2-\alpha} \frac{F^{0}}{D},
\end{gathered}
$$

where

$$
F^{0}=C_{1}^{F}\left\|\partial_{x} c_{0}^{e f f}\right\|_{L^{2}((0,+\infty) \times(0, T))}+C_{2}^{F} k \leq C_{3}^{F} \varepsilon^{-\alpha / 4} .
$$

Proof. We are in the situation of Proposition 1 with $\xi_{0}^{\varepsilon}=0$ and $g^{\varepsilon}=k c_{0}^{\text {eff }}$. Consequently, for $\xi=c^{\varepsilon}-c_{0}^{e f f}$ we have

$$
\begin{aligned}
& \mathcal{E}(\xi, t) \leq k \int_{0}^{t} \int_{0}^{+\infty} c_{0}^{e f f}\left(\int_{0}^{1} c^{\varepsilon} d y-\left.c^{\varepsilon}\right|_{y=1}\right) \Psi^{2} d x d \tau+2 D \varepsilon^{\alpha} \\
& \int_{0}^{t} \int_{\Omega^{+}}|\xi|^{2} \Psi^{2}(x) d x d y d \tau-\int_{0}^{t} \int_{\Omega^{+}} Q\left(\frac{1}{3}-y^{2}\right) \xi \partial_{x} c_{0}^{e f f} \Psi^{2} d x d y d \tau .
\end{aligned}
$$

It remains to estimate the first and third terms on the right. We have

$$
\begin{aligned}
& \left|\int_{0}^{t} \int_{\Omega^{+}} Q \partial_{x} c_{0}^{e f f}\left(\frac{1}{3}-y^{2}\right) \xi \Psi^{2}(x) d x d y d \tau\right| \\
= & \left|\int_{0}^{t} \int_{\Omega^{+}} Q \partial_{x} c_{0}^{e f f}\left(\frac{y}{3}-\frac{y^{3}}{3}\right) \partial_{y} \xi \Psi^{2}(x) d x d y d \tau\right|
\end{aligned}
$$

and

$$
\begin{gathered}
k\left|\int_{0}^{t} \int_{0}^{+\infty} c_{0}^{e f f}\left(\int_{0}^{1} \xi d y-\left.\xi\right|_{y=1}\right) \Psi^{2} d x d \tau\right| \\
\leq \frac{D}{8} \varepsilon^{\alpha} \int_{0}^{t} \int_{\Omega^{+}} \Psi^{2}(x)\left|\partial_{y} \xi\right|^{2} d x d y d \tau+\frac{k^{2}}{D} \varepsilon^{2-\alpha} \int_{0}^{t} \int_{0}^{+\infty}\left(c_{0}^{e f f}\right)^{2} \Psi^{2} d x d \tau .
\end{gathered}
$$

Inserting (54)-(55) into (53) gives

$$
\begin{aligned}
\mathcal{E}\left(c^{\varepsilon}-c_{0}^{e f f}, t\right) & \leq \varepsilon^{2-\alpha} \int_{0}^{t} \int_{0}^{+\infty}\left\{\frac{2 k^{2}}{D}\left(c_{0}^{e f f}\right)^{2}+\frac{32}{315} \frac{Q^{2}}{D}\left(\partial_{x} c_{0}^{e f f}\right)^{2}\right\} \Psi^{2} d x d \tau \\
& +\int_{0}^{t} \int_{0}^{1} \int_{0}^{+\infty} 2 D \varepsilon^{\alpha} \Psi^{2}(x)\left(c^{\varepsilon}-c_{0}^{e f f}\right)^{2} d x d y d \tau
\end{aligned}
$$


and applying Gronwall's inequality results in (49)-(51).

Corollary 2. Let $\Omega_{K}=(0, K) \times(0,1), K>0$. Then we have

$$
\left\|c^{\varepsilon}-c_{0}^{e f f}\right\|_{L^{\infty}\left(0, T ; L^{2}\left(\Omega_{K}\right)\right)} \leq C \varepsilon^{1-3 \alpha / 4} .
$$

Remark 1. It is reasonable to expect $L^{1}$ estimates with better powers for $\varepsilon$. Unfortunately, testing (40) by the regularized sign $\left(c^{\varepsilon}-c_{0}^{e f f}\right)$ does not lead to a useful result. Hence at this stage claiming a $\sqrt{\varepsilon}$ estimate in $L^{1}$ is not justified.

Remark 2. There are results by Grenier [7] and Grenier and Guès [8] on singular perturbation problems. In [7] Grenier supposes that $q$ is smooth and zero at $x=0$, together with its derivatives. Such hypotheses allow better estimates.

Remark 3. For $\alpha=1$, the estimates (24) and (57) imply that the functions $\exp \{-k t\} \chi_{\{x>Q t\}}$ and $c_{0}^{e f f}$ approximate $c^{\varepsilon}$ in $L^{\infty}\left(L^{2}\right)$ with the same order given by $C \varepsilon^{1 / 4}$.

Remark 4. Estimate (57) is not useful when $\alpha>4 / 3$.

4. The formal 2-scale expansion leading to Taylor's dispersion. The estimate obtained in the previous section is not satisfactory. However, it is known that the Taylor dispersion model gives a very good $1 \mathrm{D}$ approximation. This motivates us to derive higher precision approximations. We give a formal 2-scale asymptotic expansion to obtain Taylor's (including the chemistry) dispersion formula.

We start with the problem (35)-(39) and search for $c^{\varepsilon}$ in the form

$$
c^{\varepsilon}=c^{0}(x, t ; \varepsilon)+\varepsilon c^{1}(x, y, t)+\varepsilon^{2} c^{2}(x, y, t)+\cdots .
$$

After introducing (58) into (35) we get

$$
\begin{gathered}
\varepsilon^{0}\left\{\partial_{t} c^{0}+Q\left(1-y^{2}\right) \partial_{x} c^{0}-D \varepsilon^{\alpha-1} \partial_{y y} c^{1}\right\} \\
+\varepsilon\left\{\partial_{t} c^{1}+Q\left(1-y^{2}\right) \partial_{x} c^{1}-D \varepsilon^{\alpha-1} \partial_{x x} c^{0}-D \varepsilon^{\alpha-1} \partial_{y y} c^{2}\right\}=\mathcal{O}\left(\varepsilon^{2}\right) .
\end{gathered}
$$

To satisfy (59) for every $\varepsilon \in\left(0, \varepsilon_{0}\right)$, all coefficients in front of the powers of $\varepsilon$ should be zero.

The problem corresponding to the $\varepsilon^{0}$ is

$$
\left\{\begin{array}{l}
-D \partial_{y y} c^{1}=-\varepsilon^{1-\alpha} Q\left(\frac{1}{3}-y^{2}\right) \partial_{x} c^{0}-\varepsilon^{1-\alpha}\left(\partial_{t} c^{0}+2 Q \partial_{x} c^{0} / 3\right) \text { on }(0,1), \\
\partial_{y} c^{1}=0 \text { on } y=0 \text { and }-D \partial_{y} c^{1}=k \varepsilon^{1-\alpha} c^{0} \text { on } y=1
\end{array}\right.
$$

for every $(x, t) \in(0,+\infty) \times(0, T)$. By Fredholm's alternative, this problem has a solution if and only if

$$
\partial_{t} c^{0}+\frac{2 Q \partial_{x} c^{0}}{3}+k c^{0}=0 \quad \text { in }(0, L) \times(0, T) .
$$

Unfortunately our initial and boundary data are incompatible, and therefore the solution to this hyperbolic equation is discontinuous. Since the asymptotic expansion for $c^{\varepsilon}$ involves derivatives of $c^{0}$, (61) does not suit our needs. In [3] this difficulty was overcome by assuming compatible initial and boundary data. We proceed by following an idea from [18] and suppose that

$$
\partial_{t} c^{0}+\frac{2 Q \partial_{x} c^{0}}{3}+k c^{0}=\mathcal{O}(\varepsilon) \quad \text { in }(0,+\infty) \times(0, T) .
$$


This hypothesis will be justified a posteriori, after getting an equation for $c^{0}$.

Combining (60) and (61) and using hypothesis (62) leads us to consider

$$
\left\{\begin{array}{l}
-D \partial_{y y} c^{1}=-\varepsilon^{1-\alpha} Q\left(\frac{1}{3}-y^{2}\right) \partial_{x} c^{0}+\varepsilon^{1-\alpha} k c^{0} \text { on }(0,1), \\
\partial_{y} c^{1}=0 \text { on } y=0 \text { and }-D \partial_{y} c^{1}=k \varepsilon^{1-\alpha} c^{0} \text { on } y=1
\end{array}\right.
$$

for every $(x, t) \in(0,+\infty) \times(0, T)$. Consequently

$$
c^{1}(x, y, t)=\varepsilon^{1-\alpha}\left(\frac{Q}{D}\left(\frac{y^{2}}{6}-\frac{y^{4}}{12}\right) \partial_{x} c^{0}+\frac{k}{D}\left(\frac{1}{6}-\frac{y^{2}}{2}\right) c^{0}+C_{0}(x, t)\right),
$$

where $C_{0}$ is an arbitrary function.

The problem corresponding to $\varepsilon^{1}$ is

$$
\left\{\begin{array}{l}
-D \partial_{y y} c^{2}=D \varepsilon \partial_{x x} c^{1}-\varepsilon^{1-\alpha} Q\left(1-y^{2}\right) \partial_{x} c^{1}+D \partial_{x x} c^{0}-\varepsilon^{1-\alpha} \partial_{t} c^{1} \\
-\varepsilon^{-\alpha}\left(\partial_{t} c^{0}+\frac{2 Q \partial_{x} c^{0}}{3}+k c^{0}\right) \text { on }(0,1), \\
\partial_{y} c^{2}=0 \text { on } y=0 \text { and }-D \partial_{y} c^{2}=k \varepsilon^{1-\alpha} c^{1} \text { on } y=1
\end{array}\right.
$$

for every $(x, t) \in(0,+\infty) \times(0, T)$. This problem has a solution if and only if

$$
\partial_{t} c^{0}+\frac{2 Q \partial_{x} c^{0}}{3}+k\left(c^{0}+\left.\varepsilon c^{1}\right|_{y=1}\right)+\varepsilon \partial_{t}\left(\int_{0}^{1} c^{1} d y\right)-\varepsilon^{\alpha} D \partial_{x x} c^{0}
$$

$$
+Q \varepsilon \partial_{x}\left(\int_{0}^{1}\left(1-y^{2}\right) c^{1} d y\right)-D \varepsilon^{1+\alpha} \partial_{x x}\left(\int_{0}^{1} c^{1} d y\right)=0 \quad \text { in }(0,+\infty) \times(0, T) .
$$

Note that this is the equation for $c^{0}$. In order to get the simplest possible equation we choose $C_{0}$ such that $\int_{0}^{1} c^{1} d y=0$. This implies

$$
c^{1}(x, y, t)=\varepsilon^{1-\alpha}\left(\frac{Q}{D}\left(\frac{y^{2}}{6}-\frac{y^{4}}{12}-\frac{7}{180}\right) \partial_{x} c^{0}+\frac{k}{D}\left(\frac{1}{6}-\frac{y^{2}}{2}\right) c^{0}\right),
$$

and (66) becomes

$\partial_{t} c^{0}+Q\left(\frac{2}{3}+\frac{4 k}{45 D} \varepsilon^{2-\alpha}\right) \partial_{x} c^{0}+k\left(1-\frac{k}{3 D} \varepsilon^{2-\alpha}\right) c^{0}=\varepsilon^{\alpha} \tilde{D} \partial_{x x} c^{0} \quad$ in $\quad(0,+\infty) \times(0, T)$,

with

$$
\tilde{D}=D+\frac{8}{945} \frac{Q^{2}}{D} \varepsilon^{2(1-\alpha)} .
$$

As a result, problem (65) transforms into

$$
\left\{\begin{array}{l}
-D \partial_{y y} c^{2}=\varepsilon^{2-2 \alpha}\left\{-\frac{Q^{2}}{D} \partial_{x x} c^{0}\left\{\frac{8}{945}+\left(1-y^{2}\right)\left(\frac{y^{2}}{6}-\frac{y^{4}}{12}-\frac{7}{180}\right)\right\}\right. \\
+\partial_{x} c^{0} \frac{Q k}{D}\left\{\frac{2}{45}-\left(1-y^{2}\right)\left(\frac{1}{6}-\frac{y^{2}}{2}\right)\right\}+\frac{2 k Q}{45 D} \partial_{x} c^{0} \\
-\frac{k^{2}}{3 D} c^{0}-\left(\frac{y^{2}}{6}-\frac{y^{4}}{12}-\frac{7}{180}\right)\left(\partial_{x t} c^{0} \frac{Q}{D}-\varepsilon^{\alpha} Q \partial_{x x x} c^{0}\right) \\
\left.-\left(\frac{1}{6}-\frac{y^{2}}{2}\right)\left(\partial_{t} c^{0} \frac{k}{D}-\varepsilon^{\alpha} k \partial_{x x} c^{0}\right)\right\} \text { on }(0,1), \quad \partial_{y} c^{2}=0 \text { on } y=0, \\
\text { and }-D \partial_{y} c^{2}=\frac{Q k}{D} \varepsilon^{2-2 \alpha} \partial_{x} c^{0} \frac{2}{45}-\frac{k^{2}}{3 D} \varepsilon^{2-2 \alpha} c^{0} \text { on } y=1 .
\end{array}\right.
$$


If we choose $c^{2}$ such that $\int_{0}^{1} c^{2} d y=0$, then

$$
\begin{gathered}
c^{2}(x, y, t)=\varepsilon^{2-2 \alpha}\left\{-\frac{Q^{2}}{D^{2}} \partial_{x x} c^{0}\left(\frac{281}{453600}+\frac{23}{1512} y^{2}-\frac{37}{2160} y^{4}+\frac{1}{120} y^{6}-\frac{1}{672} y^{8}\right)\right. \\
+\left(\frac{Q}{D^{2}} \partial_{x t} c^{0}-\varepsilon^{\alpha} \frac{Q}{D} \partial_{x x x} c^{0}\right)\left(\frac{31}{7560}-\frac{7}{360} y^{2}+\frac{y^{4}}{72}-\frac{y^{6}}{360}\right) \\
+\frac{Q k}{D^{2}} \partial_{x} c^{0}\left(\frac{y^{6}}{60}-\frac{y^{4}}{18}+\frac{11 y^{2}}{180}-\frac{11}{810}\right) \\
\left.+\left(\frac{k 1)}{2 D^{2}} \partial_{t} c^{0}-\frac{k}{2 D} \varepsilon^{\alpha} \partial_{x x} c^{0}\right)\left(-\frac{y^{4}}{12}+\frac{y^{2}}{6}-\frac{7}{180}\right)+\left(\frac{Q k}{45 D^{2}} \partial_{x} c^{0}-\frac{k^{2}}{6 D^{2}} c^{0}\right)\left(\frac{1}{3}-y^{2}\right)\right\} .
\end{gathered}
$$

5. Boundary layer. The higher order approximations in the asymptotic expansion for $c^{\varepsilon}$ do not satisfy the boundary conditions. Such incompatibility suggests that we should correct them using an appropriate boundary layer:

$$
\left\{\begin{array}{l}
-\Delta_{y, z} \beta=0 \text { for }(z, y) \in \Omega^{+} \\
-\partial_{y} \beta=0 \text { for } y=1, \quad \text { and for } y=0 \\
\beta=\frac{y^{2}}{6}-\frac{y^{4}}{12}-\frac{7}{180} \text { for } z=0
\end{array}\right.
$$

Using the elementary variational theory for PDEs, we obtain the existence of a unique solution $\beta \in L_{l o c}^{2}\left(\Omega^{+}\right)$such that $\nabla \beta \in L_{l o c}^{2}\left(\Omega^{+}\right)^{2}$. Since the average of the boundary value at $z=0$ is zero, it follows that $\int_{0}^{1} \beta(z, y) d y=0$ for every $z \in(0,+\infty)$. This allows us to apply Poincaré's inequality in $H^{1}$ :

$$
\int_{0}^{1} \varphi^{2} d y \leq \frac{1}{\pi^{2}} \int_{0}^{1}\left|\partial_{y} \varphi\right|^{2} d y \forall \varphi \in H^{1}(0,1), \quad \int_{0}^{1} \varphi d y=0
$$

and conclude that in fact $\beta \in H^{1}\left(\Omega^{+}\right)$. In order to prove that $\beta$ represents a boundary layer, one should prove the exponential decay. We apply the theory from [14] and get the following result describing the decay of $\beta$ as $z \rightarrow+\infty$.

Proposition 3. There exists a constant $\gamma_{0}>0$ such that the solution $\beta$ of (72) satisfies the estimates

$$
\begin{gathered}
\int_{z}^{+\infty} \int_{0}^{1}\left|\nabla_{y, z} \beta\right|^{2} d y d z \leq c_{0} e^{-\gamma_{0} z}, \quad z>0, \\
|\beta(y, z)| \leq c_{0} e^{-\gamma_{0} z} \quad \forall(y, z) \in \Omega^{+} .
\end{gathered}
$$

6. First correction. As explained, estimate (57) is not satisfactory. To get a better approximation we consider the correction, which was constructed using the formal 2-scale expansion in section 4 . 
Let $0 \leq \alpha<2$. We start with the $\mathcal{O}\left(\varepsilon^{4-2 \alpha}\right)$ approximation and consider the function

(76)

$$
c_{1}^{e f f}(x, y, t ; \varepsilon)=c(x, t ; \varepsilon)+\varepsilon^{2-\alpha} \zeta(t)\left(\frac{Q}{D}\left(\frac{y^{2}}{6}-\frac{y^{4}}{12}-\frac{7}{180}\right) \frac{\partial c}{\partial x}+\frac{k}{D}\left(\frac{1}{6}-\frac{y^{2}}{2}\right) c\right),
$$

where $c$ is the solution to the effective problem with Taylor's dispersion coefficient including the reaction terms:

$$
\left\{\begin{array}{l}
\partial_{t} c+Q\left(\frac{2}{3}+\frac{4 k}{45 D} \varepsilon^{2-\alpha}\right) \partial_{x} c+k\left(1-\frac{k}{3 D} \varepsilon^{2-\alpha}\right) c \\
=\left(D \varepsilon^{\alpha}+\frac{8}{945} \frac{Q^{2}}{D} \varepsilon^{2-\alpha}\right) \partial_{x x} c \text { in }(0,+\infty) \times(0, T), \\
\left.c\right|_{x=0}=0,\left.c\right|_{t=0}=1, \partial_{x} c \in L^{2}((0,+\infty) \times(0, T)) .
\end{array}\right.
$$

The cut-off in time $\zeta$ is given by (26) and we use it to eliminate the time-like boundary layer appearing at $t=0$. These effects are not visible in the formal expansion.

Let $\mathcal{L}^{\varepsilon}$ be the differential operator given by (34). Following the formal expansion from section 4 , we find that $\mathcal{L}^{\varepsilon}$ applied to the correction without boundary layer functions and cut-offs would give $F_{1}^{\varepsilon}+F_{2}^{\varepsilon}+F_{3}^{\varepsilon}+F_{4}^{\varepsilon}+F_{5}^{\varepsilon}$, where

$$
\left\{\begin{array}{l}
F_{1}^{\varepsilon}=\partial_{x x} c \frac{Q^{2}}{D} \varepsilon^{2-\alpha}\left\{\frac{8}{945}+\left(1-y^{2}\right)\left(\frac{y^{2}}{6}-\frac{y^{4}}{12}-\frac{7}{180}\right)\right\} \\
F_{2}^{\varepsilon}=\partial_{x} c \frac{Q k}{D} \varepsilon^{2-\alpha}\left\{-\frac{2}{45}+\left(1-y^{2}\right)\left(\frac{1}{6}-\frac{y^{2}}{2}\right)\right\} \\
F_{3}^{\varepsilon}=\varepsilon^{2-\alpha}\left(\frac{y^{2}}{6}-\frac{y^{4}}{12}-\frac{7}{180}\right)\left\{\partial_{x t} c \frac{Q}{D}-\varepsilon^{\alpha} \partial_{x x x} c Q\right\} \\
F_{4}^{\varepsilon}=\varepsilon^{2-\alpha}\left(\frac{1}{6}-\frac{y^{2}}{2}\right)\left\{\partial_{t} c \frac{k}{D}-\varepsilon^{\alpha} \partial_{x x} c k\right\} \\
F_{5}^{\varepsilon}=\varepsilon^{2-\alpha}\left\{-\frac{2}{45} \partial_{x} c \frac{Q k}{D}+\frac{k^{2}}{3 D} c\right\} .
\end{array}\right.
$$

These functions are not integrable up to $t=0$, and for handling them we introduce the cut-off $\zeta$.

Applying $\mathcal{L}^{\varepsilon}$ to $c_{1}^{\text {eff }}$ gives

$$
\begin{gathered}
\mathcal{L}^{\varepsilon}\left(c_{1}^{\text {eff }}\right)=\zeta(t) \sum_{j=1}^{5} F_{j}^{\varepsilon}+\left(\varepsilon^{2-\alpha} \partial_{x x} c \frac{Q^{2}}{D} \frac{8}{945}+Q\left(\frac{1}{3}-y^{2}\right) \partial_{x} c-k c\right) \\
\times(1-\zeta(t))+\zeta^{\prime}(t) \varepsilon^{2-\alpha}\left(\partial_{x} c \frac{Q}{D}\left\{\frac{y^{2}}{6}-\frac{y^{4}}{12}-\frac{7}{180}\right\}+\frac{k}{2 D}\left(\frac{1}{3}-y^{2}\right) c\right) \\
=: \Phi_{1}^{\varepsilon} \text { and }-\mathcal{L}^{\varepsilon}\left(c_{1}^{\text {eff }}\right)=\mathcal{L}^{\varepsilon}\left(c^{\varepsilon}-c_{1}^{\text {eff }}\right)=-\Phi_{1}^{\varepsilon} .
\end{gathered}
$$

At the lateral boundary $y=1$ we have

$$
\begin{gathered}
-\left.D \varepsilon^{\alpha-2} \partial_{y} c_{1}^{e f f}\right|_{y=1}=\zeta(t) k c \\
\left.k c_{1}^{e f f}\right|_{y=1}=k\left(c+\varepsilon^{2-\alpha} \frac{Q}{D} \zeta(t) \frac{2}{45} \partial_{x} c-\varepsilon^{2-\alpha} \frac{k}{3 D} c \zeta(t)\right) .
\end{gathered}
$$


Now $c^{\varepsilon}-c_{1}^{\text {eff }}$ satisfies the system

$$
\begin{gathered}
\mathcal{L}^{\varepsilon}\left(c^{\varepsilon}-c_{1}^{e f f}\right)=-\Phi_{1}^{\varepsilon} \text { in } \Omega^{+} \times(0, T), \\
\left.\partial_{y}\left(c^{\varepsilon}-c_{1}^{e f f}\right)\right|_{y=0}=0 \text { on }(0,+\infty) \times(0, T), \\
-\left.D \varepsilon^{\alpha-2} \partial_{y}\left(c^{\varepsilon}-c_{1}^{e f f}\right)\right|_{y=1}=\left.k\left(c^{\varepsilon}-c_{1}^{e f f}\right)\right|_{y=1}+\left.g^{\varepsilon}\right|_{y=1} \text { on }(0,+\infty) \times(0, T), \\
\left.\left(c^{\varepsilon}-c_{1}^{e f f}\right)\right|_{t=0}=0 \text { on } \Omega^{+} \text {and }\left.\left(c^{\varepsilon}-c_{1}^{e f f}\right)\right|_{x=0}=\eta_{0}^{\varepsilon} \text { on }(0,1) \times(0, T), \\
\text { with } g^{\varepsilon}=k \zeta(t) \varepsilon^{2-\alpha}\left(\partial_{x} c \frac{2 Q}{45 D}-c \frac{k}{3 D}\right)+(1-\zeta) k c \\
\text { and } \eta_{0}^{\varepsilon}=-\left.\varepsilon^{2-\alpha} \zeta(t) \partial_{x} c\right|_{x=0}\left(\frac{y^{2}}{6}-\frac{y^{4}}{12}-\frac{7}{180}\right) \frac{Q}{D} .
\end{gathered}
$$

As a next step we estimate $\Phi_{1}^{\varepsilon}$ to find out if the right-hand side is smaller than in section 3 .

Proposition 4. Let $O_{t}=\Omega^{+} \times(0, t)$ and let $\varphi \in H^{1}\left(O_{T}\right)$ satisfy $\varphi=0$ at $x=0$. Then we have

$$
\begin{gathered}
\left|\int_{0}^{t} \int_{\Omega^{+}} \zeta F_{1}^{\varepsilon} \varphi d x d y d \tau\right| \leq C \varepsilon^{3(2-\alpha) / 2}\left\|\zeta(\tau) \partial_{x x} c\right\|_{L^{2}\left(O_{t}\right)}\left\|\varepsilon^{\alpha / 2-1} \partial_{y} \varphi\right\|_{L^{2}\left(O_{t}\right)} \\
\leq C\left(\varepsilon^{3-5 \alpha / 2} H(1-\alpha)+\varepsilon^{1-\alpha / 2} H(\alpha-1)\right)\left\|\varepsilon^{\alpha / 2-1} \partial_{y} \varphi\right\|_{L^{2}\left(O_{t}\right)} ; \\
\left|\int_{0}^{t} \int_{\Omega^{+}} \zeta(\tau) F_{3}^{\varepsilon} \varphi d x d y d \tau\right| \leq C \varepsilon^{3(2-\alpha) / 2} \\
\cdot\left(\left\|\zeta(\tau) \partial_{x t} c\right\|_{L^{2}\left(O_{t}\right)}+\left\|\zeta(\tau) \partial_{x x} c\right\|_{L^{2}\left(O_{t}\right)}\right) \cdot\left\|\varepsilon^{\alpha / 2-1} \partial_{y} \varphi\right\|_{L^{2}\left(O_{t}\right)} \\
\quad \leq C\left(\varepsilon^{3-5 \alpha / 2} H(1-\alpha)+\varepsilon^{1-\alpha / 2} H(\alpha-1)\right)\left\|\varepsilon^{\alpha / 2-1} \partial_{y} \varphi\right\|_{L^{2}\left(O_{t}\right)} ; \\
\left|\int_{0}^{t} \int_{\Omega^{+}}(1-\zeta) \partial_{x x} c \varepsilon^{2-\alpha} \frac{Q^{2}}{D} \varphi d x d y d \tau\right| \leq C \varepsilon^{2-3 \alpha / 2}\left\|\varepsilon^{\alpha / 2} \partial_{x} \varphi\right\|_{L^{2}\left(O_{t}\right)} \\
\cdot\left\|(1-\zeta) \partial_{x} c\right\|_{L^{2}\left(O_{t}\right)} \leq C \varepsilon^{2-3 \alpha / 2}\left\|\varepsilon^{\alpha / 2} \partial_{x} \varphi\right\|_{L^{2}\left(O_{t}\right)} ; \\
\int_{\Omega^{+}}(1-\zeta) Q\left(\frac{1}{3}-y^{2}\right) \partial_{x} c \varphi d x d y d \tau \mid \leq C \varepsilon^{1-\alpha / 2}\left\|\varepsilon^{\alpha / 2-1} \partial_{y} \varphi\right\|_{L^{2}\left(O_{t}\right)} \\
\cdot\left\|(1-\zeta) \partial_{x} c\right\|_{L^{2}\left(O_{t}\right)} \leq C \varepsilon^{1-\alpha / 2}\left\|\varepsilon^{\alpha / 2-1} \partial_{y} \varphi\right\|_{L^{2}\left(O_{t}\right)} ; \\
\int_{0}^{t} \int_{\Omega^{+}} \zeta^{\prime}\left(\frac{t}{D \varepsilon}\right) \varepsilon^{2-\alpha}\left\{\partial_{x} c \frac{Q}{D}\left\{\frac{y^{2}}{6}-\frac{y^{4}}{12}-\frac{7}{180}\right\}-\frac{k}{2 D}\left(\frac{1}{3}-y^{2}\right) c\right\} \cdot \varphi d x d y d \tau \mid \\
\leq C \varepsilon^{3-3 \alpha / 2}\left\|\zeta^{\prime} \partial_{x} c\right\|_{L^{2}\left(O_{t}\right)}\left\|\varepsilon^{\alpha / 2-1} \partial_{y} \varphi\right\|_{L^{2}\left(O_{t}\right)} \\
\leq C\left(\varepsilon^{3-5 \alpha / 2} H(1-\alpha)+\varepsilon^{1-\alpha / 2} H(\alpha-1)\right)\left\|\varepsilon^{\alpha / 2-1} \partial_{y} \varphi\right\|_{L^{2}\left(O_{t}\right)} \cdot
\end{gathered}
$$

Proof. First note that in (88)-(89) and (91)-(92) the averages of the polynomials in $y$ are zero. We write them in the form $P(y)=\partial_{y} P_{1}(y)$, where $P_{1}$ has zero traces at $y=0,1$. After partial integration with respect to $y$ and applying the results from section 2 , we obtain the estimates (88)-(89) and (91)-(92). Since $(1-\zeta) \partial_{x x} c$ is not square integrable, we cannot use the same approach to obtain (90). It is obtained by partial integration with respect to $x$. 
Proposition 5. With $\varphi$ as in Proposition 4 we have

$$
\begin{aligned}
& \left|\int_{0}^{t} \int_{\Omega^{+}} \zeta F_{2}^{\varepsilon} \varphi d x d y d \tau\right| \leq C \varepsilon^{3(1-\alpha / 2)}\left\|\zeta \partial_{x} c\right\|_{L^{2}\left(O_{t}\right)}\left\|\varepsilon^{\alpha / 2-1} \partial_{y} \varphi\right\|_{L^{2}\left(O_{t}\right)} \\
& \quad \leq C\left(\varepsilon^{3-7 \alpha / 4} H(1-\alpha)+\varepsilon^{5 / 2-5 \alpha / 4} H(\alpha-1)\right)\left\|\varepsilon^{\alpha / 2-1} \partial_{y} \varphi\right\|_{L^{2}\left(O_{t}\right)}
\end{aligned}
$$

$\left|\int_{0}^{t} \int_{\Omega^{+}} \zeta F_{4}^{\varepsilon} \varphi d x d y d \tau\right| \leq C \varepsilon^{3-3 \alpha / 2}\left(\left\|\zeta \partial_{t} c\right\|_{L^{2}\left(O_{t}\right)}+\varepsilon^{\alpha}\left\|\zeta \partial_{x x} c\right\|_{L^{2}\left(O_{t}\right)}\right)\left\|\varepsilon^{\alpha / 2-1} \partial_{y} \varphi\right\|_{L^{2}\left(O_{t}\right)}$

$$
\leq C\left(\varepsilon^{3-7 \alpha / 4)} H(1-\alpha)+\varepsilon^{5(2-\alpha) / 4} H(\alpha-1)\right)\left\|\varepsilon^{\alpha / 2-1} \partial_{y} \varphi\right\|_{L^{2}\left(O_{t}\right)}
$$

$$
\begin{gathered}
\left|\int_{0}^{t} \int_{0}^{+\infty} \zeta \partial_{x} c \varepsilon^{2-\alpha}\left(\int_{0}^{1} \varphi d y-\left.\varphi\right|_{y=1}\right) d x d \tau\right| \\
\leq C \varepsilon^{2-\alpha}\left\|\partial_{x} c\right\|_{L^{2}\left(0, t ; L^{2}((0,+\infty))\right)}\left\|\int_{0}^{1} \varphi d y-\left.\varphi\right|_{y=1}\right\|_{L^{2}\left(O_{t}\right)} \\
\leq C\left(\varepsilon^{3-7 \alpha / 4} H(1-\alpha)+\varepsilon^{5(2-\alpha) / 4} H(\alpha-1)\right)\left\|\varepsilon^{\alpha / 2-1} \partial_{y} \varphi\right\|_{L^{2}\left(O_{t}\right)} ;
\end{gathered}
$$

$$
\begin{gathered}
\left|\int_{0}^{t} \int_{0}^{+\infty} \zeta(t) c \varepsilon^{2-\alpha}\left(\int_{0}^{1} \varphi d y-\left.\varphi\right|_{y=1}\right) d x d \tau\right| \leq C \varepsilon^{3(1-\alpha / 2)}\left\|\varepsilon^{\alpha / 2-1} \partial_{y} \varphi\right\|_{L^{2}\left(O_{t}\right)} \\
\quad\left|\int_{0}^{t} \int_{0}^{+\infty}(1-\zeta(t)) c\left(\int_{0}^{1} \varphi d y-\left.\varphi\right|_{y=1}\right) d x d \tau\right| \\
\leq C\left(\varepsilon H(1-\alpha)+\varepsilon^{2-\alpha} H(\alpha-1)\right)\left\|\varepsilon^{\alpha / 2-1} \partial_{y} \varphi\right\|_{L^{2}\left(O_{t}\right)} .
\end{gathered}
$$

Corollary 3. With $\Phi_{1}^{\varepsilon}$ given by (79), $g^{\varepsilon}$ by (86), and with $\varphi$ as in Proposition 4 we have

$$
\leq C\left(\varepsilon^{1-\alpha / 2} H(1-\alpha)+\varepsilon^{2-3 \alpha / 2} H(\alpha-1)\right)\left\{\left\|\varepsilon^{\alpha / 2-1} \partial_{y} \varphi\right\|_{L^{2}\left(O_{t}\right)}+\left\|\varepsilon^{\alpha / 2} \partial_{x} \varphi\right\|_{L^{2}\left(O_{t}\right)}\right\} .
$$

A natural next step would be to correct $c_{1}^{e f f}$ at $x=0$ and then apply Proposition 1. Due to the presence of the term containing the first-order derivative in $x$, the boundary layer corresponding to our problem does not enter into the theory from [14]. Consequently, one should generalize it to the second-order elliptic equations with firstorder terms. The generalization in the case of periodic boundary conditions at the lateral boundary is done in [16]. To our knowledge, the generalization in the case of Neumann's boundary conditions at the lateral boundary was never published. It seems that the results from [16] apply also to this case (see [17]). In order to avoid developing a new boundary layer theory of second-order elliptic operators with important first-order terms, we simply use the boundary layer function corresponding to the Neumann problem for the Laplace operator (72). Then the transport term is ignored and a large error in the forcing term is created. The error is concentrated at small times, and by eliminating it we would obtain an appropriate estimate.

In order to use this particular point, we prove the following proposition. 
Proposition 6. Let $\Psi(x)=1 /(x+1)$ and let $g^{\varepsilon}$ and $\Phi^{\varepsilon}$ be measurable bounded functions satisfying the conditions

$$
\Psi g^{\varepsilon} \in H^{1}\left(\Omega^{+} \times(0, T)\right) \text { and } \Psi \Phi^{\varepsilon} \in L^{2}\left(\Omega^{+} \times(0, T)\right) .
$$

Furthermore, let $\xi \in L^{\infty}\left(\Omega^{+} \times(0, T)\right), \Psi \xi \in C^{0, \alpha_{0}}\left([0, T] ; L^{2}\left(\Omega^{+}\right)\right), \Psi \nabla_{x, y} \xi \in L^{2}\left(\Omega^{+} \times\right.$ $(0, T))^{2}$ be a solution of the initial/boundary problem

$$
\begin{gathered}
\mathcal{L}^{\varepsilon}(\xi)=-\Phi^{\varepsilon} \quad \text { in } \Omega^{+} \times(0, T) ;\left.\quad \partial_{y} \xi\right|_{y=0}=0 \quad \text { on }(0,+\infty) \times(0, T), \\
-\left.D \varepsilon^{\alpha-2} \partial_{y} \xi\right|_{y=1}=\left.k \xi\right|_{y=1}+\left.g^{\varepsilon}\right|_{y=1} \text { on }(0,+\infty) \times(0, T), \\
\left.\xi\right|_{t=0}=0 \text { on } \Omega^{+} \quad \text { and }\left.\quad \xi\right|_{x=0}=0 \quad \text { on }(0,1) \times(0, T) .
\end{gathered}
$$

Then we have

$$
\begin{gathered}
\mathcal{E}\left(t^{m} \xi, t\right)=t^{2 m} \int_{\Omega^{+}} \Psi(x)^{2} \xi^{2}(t) d x d y+D \varepsilon^{\alpha} \int_{0}^{t} \int_{\Omega^{+}} \Psi(x)^{2} \tau^{2 m} \\
\times\left\{\varepsilon^{-2}\left|\partial_{y} \xi\right|^{2}+\left|\partial_{x} \xi\right|^{2}\right\} d x d y d \tau+\left.k \int_{0}^{t} \int_{0}^{+\infty} \tau^{2 m} \xi^{2}\right|_{y=1} \Psi^{2}(x) d x d \tau \\
\leq\left|\int_{\Omega^{+}} \tau^{2 m} \Psi(x)^{2} \Phi^{\varepsilon} \xi d x d y d \tau\right|+\left.\left|\int_{0}^{t} \int_{0}^{+\infty} \tau^{2 m} g^{\varepsilon}\right|_{y=3 D 1} \xi\right|_{y=3 D 1} \Psi^{2}(x) d x d \tau \mid \\
+C_{2} D \varepsilon^{\alpha} \int_{0}^{t} \int_{\Omega^{+}} \tau^{2 m} \Psi(x)^{2} \xi^{2} d x d y d \tau \quad \forall m \geq 1 .
\end{gathered}
$$

Remark 5. Clearly, we want to apply this abstract result to $\xi=c^{\varepsilon}-c_{1}^{\text {eff }}$. Then $\zeta(t) \partial_{x} c$ has the required regularity, since the cut-off eliminates the singularity. With $c$ the analysis is more complicated. By direct calculation we have $\partial_{t} c \in$ $L^{q}\left(0, T ; L^{2}((0,+\infty))\right)$ for all $q \in[1,4 / 3)$ and we obtain the required Hölder regularity by the Sobolev imbedding. $\int_{0}^{A} \int_{0}^{1}|\xi(x, y, t)|^{2} d x d y$ is Hölder-continuous with some exponent $\alpha_{0}>0$ for all $A<+\infty$, independent of $\varepsilon$. In complete analogy, $c_{0}^{\text {eff }}$ defined by (33) also has the required regularity. Finally, the difference $c^{\varepsilon}-c_{0}^{\text {eff }}$ satisfies (40) and (41) and it is zero at $x=0$ and $t=0$. Then the classical parabolic regularity theory (see, e.g., [10]) implies the Hölder regularity in the time of the $L^{2}$ norm with respect to $x, y$. After combining all these results, we obtain the required regularity of $\xi$.

Proof. By the supposed Hölder continuity, there exists $t_{M} \in[0, T], t_{M}>0$, such that

$$
\frac{1}{t_{M}^{\alpha_{0}}} \int_{0}^{+\infty} \int_{0}^{1}\left|\xi\left(x, y, t_{M}\right)\right|^{2} \Psi^{2}(x) d x d y=\max _{t \in[0, T]} \frac{1}{t^{\alpha_{0}}} \int_{0}^{+\infty} \int_{0}^{1}|\xi(x, y, t)|^{2} \Psi^{2}(x) d x .
$$

Then we have

$$
\begin{gathered}
\int_{0}^{t_{M}} m \tau^{2 m-1} \int_{\Omega^{+}}|\xi|^{2} \Psi^{2}(x) d x d y d \tau \leq \int_{\Omega^{+}} \frac{|\xi|^{2}\left(t_{M}\right)}{t_{M}^{\alpha_{0}}} \Psi^{2}(x) \int_{0}^{t_{M}} m \tau^{2 m-1+\alpha_{0}} d \tau \\
=\frac{m}{2 m+\alpha_{0}} t_{M}^{2 m} \int_{\Omega^{+}}|\xi|^{2}\left(t_{M}\right) \Psi^{2}(x) d x d y
\end{gathered}
$$


and

$$
\begin{gathered}
\quad \frac{1}{2} t_{M}^{2 m} \int_{\Omega^{+}}|\xi|^{2}\left(t_{M}\right) \Psi^{2}(x) d x d y+\left.k \int_{0}^{t_{M}} \int_{0}^{+\infty} \tau^{2 m} \xi^{2}\right|_{y=1} \Psi^{2}(x) d x d \tau \\
+\int_{0}^{t_{M}} D\left(\varepsilon^{\alpha} \int_{\Omega^{+}} \tau^{2 m}\left|\partial_{x} \xi\right|^{2}(\tau) \Psi^{2}(x) d x d y+\varepsilon^{\alpha-2} \int_{\Omega^{+}} \tau^{2 m}\left|\partial_{y} \xi\right|^{2}(\tau) \Psi^{2}(x) d x d y\right) d \tau \\
\leq-\int_{0}^{t_{M}} \int_{\Omega^{+}} \tau^{2 m} \Phi^{\varepsilon} \xi d x d y d \tau-\left.\left.k \int_{0}^{t} \int_{0}^{+\infty} \tau^{2 m} \xi\right|_{y=1} g^{\varepsilon}\right|_{y=1} \Psi^{2}(x) d x d \tau \\
\quad+D \varepsilon^{\alpha} \int_{0}^{t_{M}} \int_{\Omega^{+}} \tau^{2 m} \Psi^{2}(x) \xi^{2} d x d y d \tau+m \int_{0}^{t_{M}} \int_{\Omega^{+}} \tau^{2 m-1}|\xi|^{2} \Psi^{2} d x d y d \tau .
\end{gathered}
$$

Using (105) we find (103) for $t=t_{M}$ and with $C_{2}=0$. Getting the estimate (103) for general $t \in(0, T)$ is now straightforward.

To use this estimate we should refine the estimates in Propositions 4 and 5. First, we note that estimate (29) changes to

$$
\begin{gathered}
\left\|t^{m} \partial_{t t} c\right\|_{L^{q}((0, T) \times(0,+\infty))}+\left\|t^{m} \partial_{t x} c\right\|_{L^{q}((0, T) \times(0,+\infty))}+\left\|t^{m} \partial_{x x} c\right\|_{L^{q}((0, T) \times(0,+\infty))} \\
\leq C_{q}(m)(\gamma \bar{D})^{1 /(2 q)-1} .
\end{gathered}
$$

Hence one gains $\varepsilon^{\alpha / 4}$ (respectively, $\varepsilon^{1 / 2-\alpha / 4}$ ) for the $L^{2}$ norm. In analogy with Propositions 4 and 5 we have Proposition 7 .

Proposition 7. Let $\varphi$ be as in Proposition 4 and let $m>1$. Then we have

$$
\begin{gathered}
\left|\int_{0}^{t} \int_{0}^{\infty} \int_{0}^{1} \tau^{m} \zeta F_{1}^{\varepsilon} \varphi d x d y d \tau\right| \leq C \varepsilon^{3(2-\alpha) / 2}\left\|\tau^{m} \partial_{x x} c\right\|_{L^{2}\left(O_{t}\right)}\left\|\varepsilon^{\alpha / 2-1} \partial_{y} \varphi\right\|_{L^{2}\left(O_{t}\right)} \\
\leq C\left(\varepsilon^{3-9 \alpha / 4} H(1-\alpha)+\varepsilon^{3 / 2-3 \alpha / 4} H(\alpha-1)\right)\left\|\varepsilon^{\alpha / 2-1} \partial_{y} \varphi\right\|_{L^{2}\left(O_{t}\right)} ; \\
\left|\int_{0}^{t} \int_{0}^{\infty} \int_{0}^{1} \tau^{m} \zeta F_{3}^{\varepsilon} \varphi d x d y d \tau\right| \leq C \varepsilon^{3(2-\alpha) / 2} \\
\cdot\left(\left\|\tau^{m} \partial_{x t} c\right\|_{L^{2}\left(O_{t}\right)}+\left\|\tau^{m} \partial_{x x} c\right\|_{L^{2}\left(O_{t}\right)}\right) \cdot\left\|\varepsilon^{\alpha / 2-1} \partial_{y} \varphi\right\|_{L^{2}\left(O_{t}\right)} \\
\leq C\left(\varepsilon^{3-9 \alpha / 4} H(1-\alpha)+\varepsilon^{3 / 2-3 \alpha / 4} H(\alpha-1)\right)\left\|\varepsilon^{\alpha / 2-1} \partial_{y} \varphi\right\|_{L^{2}\left(O_{t}\right)} ;
\end{gathered}
$$

$$
\left|\int_{0}^{t} \int_{\Omega^{+}} \zeta \tau^{m} F_{2}^{\varepsilon} \varphi d x d y d \tau\right| \leq C \varepsilon^{3(1-\alpha / 2)}\left\|\tau^{m} \zeta \partial_{x} c\right\|_{L^{2}\left(O_{t}\right)}\left\|\varepsilon^{\alpha / 2-1} \partial_{y} \varphi\right\|_{L^{2}\left(O_{t}\right)}
$$

$$
\begin{aligned}
& \leq C\left(\varepsilon^{3-7 \alpha / 4} H(1-\alpha)+\varepsilon^{5 / 2-5 \alpha / 4} H(\alpha-1)\right)\left\|\varepsilon^{\alpha / 2-1} \partial_{y} \varphi\right\|_{L^{2}\left(O_{t}\right)} ; \\
& \quad\left|\int_{0}^{t} \int_{\Omega^{+}} \zeta \tau^{m} F_{4}^{\varepsilon} \varphi d x d y d \tau\right| \leq C \varepsilon^{3-3 \alpha / 2} \\
& \cdot\left(\left\|\zeta \tau^{m} \partial_{t} c\right\|_{L^{2}\left(O_{t}\right)}+\varepsilon^{\alpha}\left\|\zeta \tau^{m} \partial_{x x} c\right\|_{L^{2}\left(O_{t}\right)}\right) \cdot\left\|\varepsilon^{\alpha / 2-1} \partial_{y} \varphi\right\|_{L^{2}\left(O_{t}\right)} \\
& \leq C\left(\varepsilon^{3-7 \alpha / 4)} H(1-\alpha)+\varepsilon^{5(2-\alpha) / 4} H(\alpha-1)\right)\left\|\varepsilon^{\alpha / 2-1} \partial_{y} \varphi\right\|_{L^{2}\left(O_{t}\right)} ;
\end{aligned}
$$




$$
\begin{gathered}
\left|\int_{0}^{t} \int_{0}^{+\infty} \zeta \tau^{m} \partial_{x} c \varepsilon^{2-\alpha}\left(\int_{0}^{1} \varphi d y-\left.\varphi\right|_{y=1}\right) d x d \tau\right| \\
\leq C \varepsilon^{2-\alpha}\left\|\tau^{m} \partial_{x} c\right\|_{L^{2}\left(O_{t}\right)}\left\|\int_{0}^{1} \varphi d y-\left.\varphi\right|_{y=1}\right\|_{L^{2}\left(O_{t}\right)} \\
\leq C\left(\varepsilon^{3-7 \alpha / 4} H(1-\alpha)+\varepsilon^{5(2-\alpha) / 4} H(\alpha-1)\right)\left\|\varepsilon^{\alpha / 2-1} \partial_{y} \varphi\right\|_{L^{2}\left(O_{t}\right)} ; \\
\left|\int_{0}^{t} \int_{0}^{+\infty} \zeta(t) \tau^{m} c \varepsilon^{2-\alpha}\left(\int_{0}^{1} \varphi d y-\left.\varphi\right|_{y=1}\right) d x d \tau\right| \\
\leq C \varepsilon^{3(1-\alpha / 2)}\left\|\varepsilon^{\alpha / 2-1} \partial_{y} \varphi\right\|_{L^{2}\left(O_{t}\right)} .
\end{gathered}
$$

Proof. These estimates are straightforward consequences of Propositions 4 and 5 .

We improve these results with respect to the other terms.

Without proof we state the following.

Proposition 8. Let $\varphi$ be as in Proposition 4 and let $m>1$. Then we have

$$
\begin{gathered}
\quad\left|\int_{0}^{t} \int_{0}^{\infty} \int_{0}^{1}(1-\zeta) \tau^{m} \partial_{x x} c \frac{Q^{2}}{D} \varepsilon^{2-\alpha} \varphi d x d y d \tau\right| \\
\leq C \varepsilon^{2-3 \alpha / 2}\left\|(1-\zeta) \tau^{m} \partial_{x} c\right\|_{L^{2}\left(O_{t}\right)}\left\|\varepsilon^{\alpha / 2} \partial_{x} \varphi\right\|_{L^{2}\left(O_{t}\right)} \\
\leq C\left(\varepsilon^{m \alpha+2-3 \alpha / 2} H(1-\alpha)+\varepsilon^{m(2-\alpha)+2-3 \alpha / 2} H(\alpha-1)\right)\left\|\varepsilon^{\alpha / 2} \partial_{x} \varphi\right\|_{L^{2}\left(O_{t}\right)} \\
\quad\left|\int_{0}^{t} \int_{0}^{\infty} \int_{0}^{1}(1-\zeta) \tau^{m} Q\left(\frac{1}{3}-y^{2}\right) \partial_{x} c \varphi d x d y d \tau\right| \\
\leq C \varepsilon^{1-\alpha / 2}\left\|(1-\zeta) \tau^{m} \partial_{x} c\right\|_{L^{2}\left(O_{t}\right)}\left\|\varepsilon^{\alpha / 2-1} \partial_{y} \varphi\right\|_{L^{2}\left(O_{t}\right)} \\
\leq C\left(\varepsilon^{m \alpha+1-\alpha / 2} H(1-\alpha)+\varepsilon^{m(2-\alpha)+1-\alpha / 2} H(\alpha-1)\right)\left\|\varepsilon^{\alpha / 2-1} \partial_{y} \varphi\right\|_{L^{2}\left(O_{t}\right)} ;
\end{gathered}
$$

$$
\begin{gathered}
\left|\int_{0}^{t} \int_{\Omega^{+}} \zeta^{\prime}\left(\frac{t}{D \varepsilon}\right) \tau^{m} \varepsilon^{2-\alpha}\left\{\partial_{x} c \frac{Q}{D}\left\{\frac{y^{2}}{6}-\frac{y^{4}}{12}-\frac{7}{180}\right\}-\frac{k}{2 D}\left(\frac{1}{3}-y^{2}\right) c\right\} \cdot \varphi d x d y d \tau\right| \\
\leq C \varepsilon^{3-3 \alpha / 2}\left\|\zeta^{\prime} \tau^{m} \partial_{x} c\right\|_{L^{2}\left(O_{t}\right)}\left\|\varepsilon^{\alpha / 2-1} \partial_{y} \varphi\right\|_{L^{2}\left(O_{t}\right)}
\end{gathered}
$$

$$
\leq C\left(\varepsilon^{3-3 \alpha / 2+\alpha(m-1)} H(1-\alpha)+\varepsilon^{3-3 \alpha / 2+(2-\alpha)(m-1)} H(\alpha-1)\right)\left\|\varepsilon^{\alpha / 2-1} \partial_{y} \varphi\right\|_{L^{2}\left(O_{t}\right)} .
$$

Before applying Proposition 6 and getting the final estimate, we should correct the trace at $x=0$. This is done by adding

$$
\bar{c}_{1}^{e f f}=-\varepsilon^{2-\alpha} \zeta(t) \beta^{\varepsilon} \partial_{x} c \frac{Q}{D},
$$

where $\beta^{\varepsilon}(x, y)=\beta(x / \varepsilon, y)$ is the boundary layer function given by (72). Let $g_{1}^{\varepsilon}=$ $g^{\varepsilon}-\varepsilon^{2-\alpha} \frac{k Q \zeta \partial_{x} c \beta^{\varepsilon}}{D}$. Then for $\xi^{\varepsilon}=c^{\varepsilon}-c_{1}^{e f f}-\bar{c}_{1}^{e f f}$ we have the problem 


$$
\begin{gathered}
\mathcal{L}^{\varepsilon}\left(\xi^{\varepsilon}\right)=-\Phi^{\varepsilon}=-\Phi_{1}^{\varepsilon}+\partial_{t} \zeta \varepsilon^{2-\alpha} \partial_{x} c \frac{Q}{D} \beta^{\varepsilon}+\varepsilon^{2-\alpha} \beta^{\varepsilon} \zeta(t) \\
\times\left\{\partial_{x t} c \frac{Q}{D}-\varepsilon^{\alpha} \partial_{x x x} c Q\right\}+\partial_{x} \beta^{\varepsilon} \frac{Q^{2}}{D}\left(1-y^{2}\right) \zeta \varepsilon^{2-\alpha} \partial_{x} c-\varepsilon^{2-\alpha} Q \partial_{x x} c \zeta(t) \\
\times\left(2 \varepsilon^{\alpha} \partial_{x} \beta^{\varepsilon}-\beta^{\varepsilon}\left(1-y^{2}\right) \frac{Q}{D}\right) \text { in } \Omega^{+} \times(0, T), \\
-D \varepsilon^{\alpha-2} \partial_{y} \xi^{\varepsilon}=k \xi^{\varepsilon}+g_{1}^{\varepsilon} \text { on }(0,+\infty) \times\{1\} \times(0, T), \\
\left.\partial_{y} \xi^{\varepsilon}\right|_{y=0}=0 \text { on }(0,+\infty) \times(0, T), \\
\left.\xi^{\varepsilon}\right|_{t=0}=0 \text { on } \Omega^{+} \text {and }\left.\xi^{\varepsilon}\right|_{x=0}=0 \text { on }(0,1) \times(0, T) .
\end{gathered}
$$

We need an estimate for the new terms. It will be obtained from the following auxiliary result.

LEMma 5. With $\beta$ and $c$ defined by (72) and (77), respectively, and with $m \geq 1$, we have

$$
\left\|\left.\tau^{m} \zeta \beta^{\varepsilon}\right|_{y=1} \partial_{x} c\right\|_{L^{2}\left(O_{t}\right)} \leq C \varepsilon^{m+1 / 4}\left\{\varepsilon^{-\alpha / 4} H(1-\alpha)+\varepsilon^{\alpha / 4-1 / 2} H(\alpha-1)\right\} \leq C \varepsilon^{m} ;
$$

$$
\left\|\tau^{m} \zeta \partial_{x} \beta^{\varepsilon} \partial_{x} c\right\|_{L^{2}\left(O_{t}\right)} \leq C \varepsilon^{m-3 / 4}\left\{\varepsilon^{-\alpha / 4} H(1-\alpha)+\varepsilon^{\alpha / 4-1 / 2} H(\alpha-1)\right\} \leq C \varepsilon^{m-1} ;
$$

$$
\begin{gathered}
\left\|\tau^{m} \zeta \partial_{x} \beta^{\varepsilon} \partial_{t} c\right\|_{L^{2}\left(O_{t}\right)} \leq C \varepsilon^{m-5 / 4}\left\{\varepsilon^{\alpha / 2} H(1-\alpha)+\varepsilon^{1-\alpha / 2} H(\alpha-1)\right\} \leq C \varepsilon^{m-5 / 4} ; \\
\left\|\tau^{m} \zeta \beta^{\varepsilon} \partial_{x x} c\right\|_{L^{2}\left(O_{t}\right)} \\
\leq C \varepsilon^{m}\left\{\left(\varepsilon^{-1 / 4-\alpha / 2}+\varepsilon^{1 / 4-3 \alpha / 4}\right) H(1-\alpha)+\left(\varepsilon^{\alpha / 2-5 / 4}+\varepsilon^{-5 / 2+3 \alpha / 4}\right) H(\alpha-1)\right\} \\
\leq C \varepsilon^{m-7 / 4} ; \\
\left\|\tau^{m} \zeta \partial_{x} \beta^{\varepsilon} \partial_{x x} c\right\|_{L^{2}\left(O_{t}\right)} \\
\leq C \varepsilon^{m-1}\left\{\left(\varepsilon^{-1 / 4-\alpha / 2}+\varepsilon^{1 / 4-3 \alpha / 4}\right) H(1-\alpha)+\left(\varepsilon^{\alpha / 2-5 / 4}+\varepsilon^{-5 / 2+3 \alpha / 4}\right) H(\alpha-1)\right\} \\
(127) \\
\leq C \varepsilon^{m-7 / 4} .
\end{gathered}
$$

Proof. Since

$$
\begin{aligned}
& \int_{0}^{+\infty}\left|\partial_{x} c \beta^{\varepsilon}\right|^{2} d \leq C \int_{0}^{+\infty} \exp \left\{-\frac{2 \gamma_{0} x}{\varepsilon}\right\} \exp \left\{-\frac{(x-\tau \bar{Q})^{2}}{2 \gamma \bar{D} \tau}\right\} \frac{d x}{\gamma \tau \bar{D}} \\
& \leq C(\varepsilon D \tau)^{-1 / 2} \exp \left\{-C_{0} \tau / \varepsilon\right\} d x d \tau,
\end{aligned}
$$

inequalities (122), (123), and (124) follow by integration with respect to $\tau$. Furthermore, since

$$
\begin{aligned}
\int_{0}^{+\infty}\left|\partial_{t} c \beta^{\varepsilon}\right|^{2} d x & \leq C \int_{0}^{+\infty} x^{2} \exp \left\{-\frac{2 \gamma_{0} x}{\varepsilon}\right\} \exp \left\{-\frac{(x-\tau \bar{Q})^{2}}{2 \gamma \bar{D} \tau^{3}}\right\} \frac{d x}{\gamma \tau \bar{D}} \\
& \leq C\left(\varepsilon D \tau^{3}\right)^{-1 / 2} \exp \left\{-C_{0} \tau / \varepsilon\right\} d x d \tau,
\end{aligned}
$$


inequality (125) follows. Finally, since

$$
\begin{gathered}
\left\|\tau^{m} \zeta \beta^{\varepsilon} \partial_{x x} c\right\|_{L^{2}\left(O_{t}\right)} \\
(130) \leq C\left(\left\|\tau^{m} \zeta \beta^{\varepsilon} \partial_{x} c\right\|_{L^{2}\left(O_{t}\right)}+\left\|\tau^{m} \zeta \beta^{\varepsilon} \partial_{t} c\right\|_{L^{2}\left(O_{t}\right)}\right)\left(\varepsilon^{-\alpha} H(1-\alpha)+\varepsilon^{\alpha-2} H(\alpha-1)\right),
\end{gathered}
$$

we obtain (126) and (127).

Proposition 9. With $\varphi$ as in Proposition 4, we have

$$
\begin{gathered}
\left|\int_{0}^{t} \int_{\Omega^{+}} \varepsilon^{2-\alpha} \tau^{m} \zeta(\tau) \beta^{\varepsilon}\left\{\partial_{x t} c \frac{Q}{D}-\varepsilon^{\alpha} \partial_{x x x} c Q\right\} \varphi d x d y d \tau\right| \\
\leq C \varepsilon^{2-\alpha}\left(\left\{\left\|\zeta \tau^{m} \partial_{t} c \partial_{x} \beta^{\varepsilon}\right\|_{L^{2}\left(O_{t}\right)}+\varepsilon^{\alpha}\left\|\tau^{m} \zeta \partial_{x} \beta^{\varepsilon} \partial_{x x} c\right\|_{L^{2}\left(O_{t}\right)}\right\}\|\varphi\|_{L^{2}\left(O_{t}\right)}\right. \\
\left.+\varepsilon^{-\alpha / 2}\left\{\left\|\zeta \tau^{m} \partial_{t} c \beta^{\varepsilon}\right\|_{L^{2}\left(O_{t}\right)}+\varepsilon^{\alpha}\left\|\tau^{m} \zeta \partial_{x x} c\right\|_{L^{2}\left(O_{t}\right)}\right\}\left\|\varepsilon^{\alpha / 2} \partial_{x} \varphi\right\|_{L^{2}\left(O_{t}\right)}\right) \\
\leq C \varepsilon^{m+1 / 4-\alpha}\left(\|\varphi\|_{L^{2}\left(O_{t}\right)}+\left\|\varepsilon^{\alpha / 2} \partial_{x} \varphi\right\|_{L^{2}\left(O_{t}\right)}\right) ; \\
\left.\mid \int_{0}^{t} \int_{\Omega^{+}} \varepsilon^{2-\alpha} \zeta \tau^{m} \partial_{x x} c \varphi\left(-\beta^{\varepsilon} \frac{Q}{D}\left(1-y^{2}\right)+2 \varepsilon^{\alpha} \partial_{x} \beta^{\varepsilon}\right)\right) d x d y d \tau \mid \\
\leq C \varepsilon^{2-\alpha}\left(\left\|\tau^{m} \zeta \partial_{x} \beta^{\varepsilon} \partial_{x x} c\right\|_{L^{2}\left(O_{t}\right)}+\left\|\tau^{m} \zeta \partial_{x} \beta^{\varepsilon} \partial_{x x} c\right\|_{L^{2}\left(O_{t}\right)}\right)\|\varphi\|_{L^{2}\left(O_{t}\right)} \\
\leq C \varepsilon^{m-\alpha+1 / 4}\|\varphi\|_{L^{2}\left(O_{t}\right)} ; \\
\leq C \varepsilon^{2-\alpha}\left\|\tau^{m} \zeta \partial_{x} \beta^{\varepsilon} \partial_{x} c\right\|_{L^{2}\left(O_{t}\right)}\|\varphi\|_{L^{2}\left(O_{t}\right)} \leq C \varepsilon^{m-\alpha+1}\|\varphi\|_{L^{2}\left(O_{t}\right)} ; \\
\left.\left|\int_{0}^{t} \int_{0}^{+\infty} \varepsilon^{2-\alpha} \zeta \tau^{m} \partial_{x} c \varphi\right|_{y=1} \beta^{\varepsilon}\right|_{y=1} d x d \tau \mid \\
\leq C \varepsilon^{2-\alpha}\left\|\tau^{m} \zeta \partial_{x} \beta^{\varepsilon} \partial_{x} c\right\|_{L^{2}\left(O_{t}\right)}\|\varphi\|_{L^{2}\left((0, t) \times \Gamma^{+}\right)} \leq C \varepsilon^{m-\alpha+1}\|\varphi\|_{L^{2}\left((0, t) \times \Gamma^{+}\right)} ; \\
\left|\int_{0}^{t} \int_{\Omega^{+}} \varepsilon^{2-\alpha} \zeta^{\prime}(\tau) \tau^{m} \partial_{x} c \varphi \beta^{\varepsilon} d x d y d \tau\right| \\
\leq C \varepsilon^{2-\alpha}\left\|\tau^{m} \zeta^{\prime} \beta^{\varepsilon} \partial_{x} c\right\|_{L^{2}\left(O_{t}\right)}\|\varphi\|_{L^{2}\left(O_{t}\right)} \leq C \varepsilon^{m-\alpha+3 / 4}\|\varphi\|_{L^{2}\left(O_{t}\right)} .
\end{gathered}
$$

At this point the application of Proposition 6 is straightforward. As a result we get the following.

THEOREM 4. Let $c$ be the solution of (77) and let $c_{1}^{\text {eff }}$ and $\bar{c}_{1}^{\text {eff }}$ be given by (76) and (117), respectively. With $\Omega_{K}=(0, K) \times(0,1), K>0$, we have

$$
\begin{gathered}
\left\|t^{3}\left(c^{\varepsilon}-c_{1}^{e f f}(x, t ; \varepsilon)-\bar{c}_{1}^{e f f}\right)\right\|_{L^{\infty}\left(0, T ; L^{2}\left(\Omega_{K}\right)\right)} \\
\leq C\left(\varepsilon^{3-9 \alpha / 4} H(1-\alpha)+\varepsilon^{3(1-\alpha / 2) / 2} H(\alpha-1)\right) ; \\
\left\|t^{3} \partial_{y}\left(c^{\varepsilon}-c_{1}^{e f f}(x, t ; \varepsilon)-\bar{c}_{1}^{e f f}\right)\right\|_{L^{2}\left(\Omega_{K} \times(0, T)\right)} \\
\leq C \varepsilon^{1-\alpha / 2}\left(\varepsilon^{3-9 \alpha / 4} H(1-\alpha)+\varepsilon^{3(1-\alpha / 2) / 2} H(\alpha-1)\right) ; \\
\left\|t^{2} \partial_{x}\left(c^{\varepsilon}-c_{1}^{e f f}(x, t ; \varepsilon)-\bar{c}_{1}^{e f f}\right)\right\|_{L^{2}\left(\Omega_{K} \times(0, T)\right)} \\
\leq C \varepsilon^{-\alpha / 2}\left(\varepsilon^{3-9 \alpha / 4} H(1-\alpha)+\varepsilon^{3(1-\alpha / 2) / 2} H(\alpha-1)\right) .
\end{gathered}
$$


7. Error estimate involving the second order in expansion. The most important power in the Peclet number $\alpha$ is $\alpha=1$ because it describes Taylor's scaling. In this case our approximation is of order $\varepsilon^{3 / 4}$ in $L^{2}$. It is of interest to reach the order $\varepsilon$ at least in this case. Also, it is of interest to get the higher order estimates because $\varepsilon$ is frequently not very small.

After the results of section 6, the leading order terms in the estimates are $\zeta F_{1}^{\varepsilon}$ and $\zeta F_{3}^{\varepsilon}$, where $F_{j}^{\varepsilon}, j=1, \ldots, 3$, are defined in (78). When deriving formally the effective equation, we have seen that they could be eliminated by introducing the next order correction. Following the formal expansion, we find that $c_{1}^{\text {eff }}$ should be replaced with $c_{1}^{\text {eff }}+c_{2}^{\text {eff }}$, where

$$
\begin{aligned}
c_{2}^{e f f}=-\varepsilon^{4-2 \alpha} \frac{Q}{D^{2}} \zeta(t)\left\{Q \partial_{x x} c(\right. & \left.\frac{281}{453600}+\frac{23}{1512} y^{2}-\frac{37}{2160} y^{4}+\frac{1}{120} y^{6}-\frac{1}{672} y^{8}-\tilde{\beta}_{1}\right) \\
\left.-\left(\partial_{x t} c-D \varepsilon^{\alpha} \partial_{x x x} c\right)\left(-\frac{1}{360} y^{6}+\frac{1}{72} y^{4}-\frac{7}{360} y^{2}-\frac{31}{7560}-\tilde{\beta}_{2}\right)\right\} & \\
+\varepsilon^{4-2 \alpha} \frac{k}{D^{2}} \zeta(t)\{ & Q \partial_{x} c\left(\frac{1}{60} y^{6}-\frac{1}{18} y^{4}+\frac{11}{180} y^{2}-\frac{11}{810}-\tilde{\beta}_{3}\right) \\
+ & \frac{1}{2}\left(\partial_{t} c-D \varepsilon^{\alpha} \partial_{x x} c\right)\left(-\frac{1}{12} y^{4}+\frac{1}{6} y^{2}-\frac{7}{180}-\tilde{\beta}_{5}\right) \\
+ & \left.\frac{Q}{45} \partial_{x} c\left(\frac{1}{3}-y^{2}-\tilde{\beta}_{4}\right)-\frac{k}{6} c\left(\frac{1}{3}-y^{2}\right)\right\},
\end{aligned}
$$

where $\tilde{\beta}_{j}, j=1, \ldots, 5$, are boundary layers analogous to (72).

The application of this additional correction term gives the following.

THEOREM 5. With the notation of Theorem 4, we have

$$
\begin{gathered}
\left\|t^{5}\left(c^{\varepsilon}-c_{1}^{e f f}(x, t ; \varepsilon)-\bar{c}_{1}^{e f f}\right)\right\|_{L^{\infty}\left(0, T ; L^{2}\left(\Omega_{K}\right)\right)} \\
\leq C\left(\varepsilon^{4-13 \alpha / 4} H(1-\alpha)+\varepsilon^{3(1-\alpha / 2) / 2} H(\alpha-1)\right) ; \\
\left\|t^{5} \partial_{y}\left(c^{\varepsilon}-c_{1}^{e f f}(x, t ; \varepsilon)-\bar{c}_{1}^{e f f}\right)\right\|_{L^{2}\left(0, T ; L^{2}\left(\Omega_{K}\right)\right)} \\
\leq C \varepsilon^{1-\alpha / 2}\left(\varepsilon^{4-13 \alpha / 4} H(1-\alpha)+\varepsilon^{3(1-\alpha / 2) / 2} H(\alpha-1)\right) ; \\
\left\|t^{5} \partial_{x}\left(c^{\varepsilon}-c_{1}^{e f f}(x, t ; \varepsilon)-\bar{c}_{1}^{e f f}\right)\right\|_{L^{2}\left(0, T ; L^{2}\left(\Omega_{K}\right)\right)} \\
\leq C \varepsilon^{-\alpha / 2}\left(\varepsilon^{4-13 \alpha / 4} H(1-\alpha)+\varepsilon^{3(1-\alpha / 2) / 2} H(\alpha-1)\right) .
\end{gathered}
$$

Proof. Applying the operator $\mathcal{L}^{\varepsilon}$, given by $(34)$, to $c^{\varepsilon}-c_{1}^{\text {eff }}-\bar{c}_{1}^{\text {eff }}-c_{2}^{\text {eff }}$ we obtain a forcing term $\Phi_{2}^{\varepsilon}$, analogous to $\Phi^{\varepsilon}$, given by (118). To control its behavior we are going to study the expression $\zeta \sum_{j=1}^{5} F_{j}$. As we have seen in Proposition 8, Lemma 5, and Proposition 9, other terms are small. We have the following:

- $F_{1}^{\varepsilon}$ and $F_{3}^{\varepsilon}$ are replaced with $\tilde{F}_{1}^{\varepsilon}$ and $\tilde{F}_{3}^{\varepsilon}$, given by

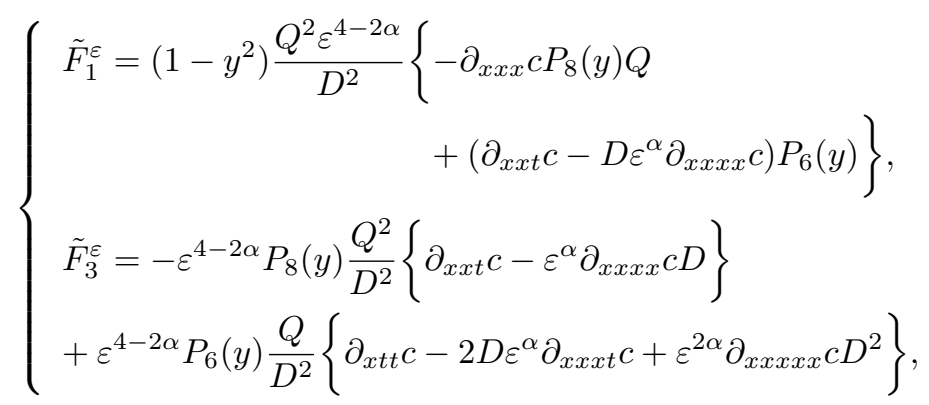


where

$$
\left\{\begin{array}{l}
P_{8}(y)=\frac{281}{453600}+\frac{23}{1512} y^{2}-\frac{37}{2160} y^{4}+\frac{1}{120} y^{6}-\frac{1}{672} y^{8} \\
P_{4}(y)=\frac{y^{2}}{6}-\frac{y^{4}}{12}-\frac{7}{180} ; P_{6}(y)=\frac{y^{3}}{18}-\frac{y^{5}}{60}-\frac{7 y}{180}-\frac{31}{7560} .
\end{array}\right.
$$

Using (32) we find, analogous to (108)-(109), that

$$
\begin{gathered}
\int_{0}^{t} \int_{0}^{\infty} \int_{0}^{1} \tau^{m} \zeta\left(\left|\tilde{F}_{1}^{\varepsilon}\right|+\left|\tilde{F}_{3}^{\varepsilon}\right|\right)|\varphi| d x d y d \tau \\
\leq C\left(\varepsilon^{4-13 \alpha / 4} H(1-\alpha)+\varepsilon^{3 / 2-3 \alpha / 4} H(\alpha-1)\right)\|\varphi\|_{L^{2}\left(O_{t}\right)}
\end{gathered}
$$

for all $\varphi \in H^{1}\left(O_{T}\right), \varphi=0$ at $x=0$ and $m>2$.

- $F_{2}^{\varepsilon}$ and $F_{4}^{\varepsilon}$ are replaced with

$$
\left\{\begin{array}{l}
\tilde{F}_{2}^{\varepsilon}=\left(1-y^{2}\right) \frac{Q k \varepsilon^{4-2 \alpha}}{D}\left\{\partial_{x x} c \frac{Q}{D} \tilde{P}_{6}(y)\right. \\
\left.+\left(\partial_{x t} c \frac{1}{2 D}-\varepsilon^{\alpha} \partial_{x x x} c \frac{1}{2}\right) P_{4}(y)+\left(\frac{Q}{45 D} \partial_{x} c-\frac{k}{6 D} c\right) P_{2}(y)\right\} \\
\tilde{F}_{4}^{\varepsilon}=-\varepsilon^{4-2 \alpha} \tilde{P}_{6}(y) \frac{Q k}{D^{2}}\left\{\partial_{x t} c-\varepsilon^{\alpha} \partial_{x x x} c D\right\} \\
+\varepsilon^{4-2 \alpha} P_{4}(y) \frac{k}{2 D^{2}}\left\{\partial_{t t} c-2 D \varepsilon^{\alpha} \partial_{x x t} c+D^{2} \varepsilon^{2 \alpha} \partial_{x x x x} c\right\} \\
+\varepsilon^{4-2 \alpha} P_{2}(y) \frac{k}{3 D^{2}}\left\{\frac{Q}{15} \partial_{x t} c-\frac{k}{2} \partial_{t} c\right. \\
\left.-\frac{D Q \varepsilon^{\alpha}}{15} \partial_{x x x} c+\frac{D k \varepsilon^{\alpha}}{2} \partial_{x x} c\right\}
\end{array}\right.
$$

where $P_{2}(y)=\frac{1}{3}-y^{2}$ and $\tilde{P}_{6}=\frac{y^{6}}{60}-\frac{y^{4}}{18}+\frac{11 y^{2}}{180}-\frac{11}{810}$. Using (32) we find, analogous to (110)-(111), that

$$
\begin{gathered}
\int_{0}^{t} \int_{0}^{\infty} \int_{0}^{1} \tau^{m} \zeta\left(\left|\tilde{F}_{2}^{\varepsilon}\right|+\left|\tilde{F}_{4}^{\varepsilon}\right|\right)|\varphi| d x d y d \tau \\
\leq C\left(\varepsilon^{4-11 \alpha / 4} H(1-\alpha)+\varepsilon^{5 / 2-5 \alpha / 4} H(\alpha-1)\right)\|\varphi\|_{L^{2}\left(O_{t}\right)}
\end{gathered}
$$

for all $\varphi \in H^{1}\left(O_{T}\right), \varphi=0$ at $x=0$ and $m>2$.

- It should be noted that the means of the polynomials in $y$, contained in $\tilde{F}_{1}$ and $\tilde{F}_{3}$, are no longer zero. Hence we cannot gain powers of $\varepsilon$ using the derivative with respect to $y$ of the test function.

- $F_{5}$ and the boundary term $k \zeta(t) \varepsilon^{2-\alpha}\left(\partial_{x} c \frac{2 Q}{45 D}-c \frac{k}{3 D}\right)$ are canceled. At the boundary $y=1$ we have a new nonhomogeneous term

$$
\hat{g}^{\varepsilon}=(1-\zeta) k c-\zeta \varepsilon^{4-2 \alpha}\left(\left.\frac{2 Q k^{2}}{45 D^{2}} \partial_{x} c \tilde{P}_{6}\right|_{y=1}+\left.\left(\frac{k}{2 D^{2}} \partial_{t} c-\varepsilon^{\alpha} \frac{k}{2 D} \partial_{x x} c\right) P_{4}\right|_{y=1}\right),
$$


and the principal boundary contribution is given by

$$
\begin{gathered}
\mid \int_{0}^{t} \int_{0}^{\infty} \int_{0}^{1} \tau^{m} \zeta \varepsilon^{4-2 \alpha}\left(\left.\frac{2 Q k^{2}}{45 D^{2}} \partial_{x} c \tilde{P}_{6}\right|_{y=1}\right. \\
\left.+\left.\left(\frac{k}{2 D^{2}} \partial_{t} c-\varepsilon^{\alpha} \frac{k}{2 D} \partial_{x x} c\right) P_{4}\right|_{y=1}\right)\left.\varphi\right|_{y=1} d x d y d \tau \mid \\
(148) \leq C\left(\varepsilon^{4-9 \alpha / 4} H(1-\alpha)+\varepsilon^{7 / 2-7 \alpha / 4} H(\alpha-1)\right)\left\|\left.\varphi\right|_{y=1}\right\|_{L^{2}((0, t) \times(0,+\infty))} .
\end{gathered}
$$

- Other terms are much smaller and do not have to be discussed.

After collecting the powers of $\varepsilon$ and applying Proposition 6 we obtain the estimates (140)-(142).

TheOREM 6. Let $c_{2}^{\text {eff }}$ be given by (139). Then, with the notation of Theorem 4, we have

$$
\begin{aligned}
& \left\|t^{5}\left(c^{\varepsilon}-c_{1}^{e f f}(x, t ; \varepsilon)-\bar{c}_{1}^{e f f}-c_{2}^{e f f}\right)\right\|_{L^{\infty}\left(0, T ; L^{1}\left(\Omega_{K}\right)\right)} \\
& \leq C\left(\varepsilon^{4-3 \alpha} H(1-\alpha)+\varepsilon^{2-\alpha} H(\alpha-1)\right), \\
& \left\|t^{5}\left(c^{\varepsilon}-c_{1}^{e f f}(x, t ; \varepsilon)-\bar{c}_{1}^{e f f}-c_{2}^{e f f}\right)\right\|_{L^{2}\left(0, T ; L^{2}\left(\Omega_{K}\right)\right)} \\
& \leq C\left(\varepsilon^{4-3 \alpha} H(1-\alpha)+\varepsilon^{2-\alpha} H(\alpha-1)\right) .
\end{aligned}
$$

Proof. First we prove the $L^{\infty}\left(L^{1}\right)$ estimate (149). We test the equation for $\xi=c^{\varepsilon}-c_{1}^{e f f}(x, t ; \varepsilon)-\bar{c}_{1}^{e f f}-c_{2}^{\text {eff }}$ by a regularized sign of $\xi$, multiplied by $\Psi^{2}$, and get

$$
\begin{gathered}
t^{m} \int_{\Omega^{+}} \Psi(x)^{2}|\xi|(t) d x d y+k \int_{0}^{t} \int_{0}^{+\infty} \tau^{m}|\xi|_{y=1} \mid \Psi^{2}(x) d x d \tau \\
\leq C_{1} \int_{0}^{t} \int_{\Omega^{+}} \tau^{m} \Psi(x)^{2}\left|\Phi_{2}^{\varepsilon}\right| d x d y d \tau+\int_{0}^{t} \int_{0}^{+\infty} \tau^{m}\left|\hat{g}^{\varepsilon}\right|_{y=1}\left|\Psi^{2}(x) d x d \tau\right| \\
+C_{2} \varepsilon^{\alpha} \int_{0}^{t} \int_{\Omega^{+}} \tau^{m} \Psi(x)^{2}|\xi| d x d y d \tau+m \int_{0}^{t} \int_{\Omega^{+}} \tau^{m-1}|\xi| \Psi^{2} d x d y d \tau
\end{gathered}
$$

for all $m \geq 3$. As before, the $L^{1}$ norm of $\Psi^{2} \xi$ is Hölder-continuous in time with some exponent $\alpha_{0}>0$. Arguing as in the proof of Proposition 6, we obtain

$$
\sup _{0 \leq t \leq T}\left\|t^{m} \Psi^{2} \xi(t)\right\|_{L^{1}\left(\Omega^{+}\right)} \leq C\left(\left\|\Psi^{2} \Phi_{2}^{\varepsilon}\right\|_{L^{1}\left(\Omega^{+} \times(0, T)\right)}+\left\|\left.\Psi^{2} \hat{g}^{\varepsilon}\right|_{y=1}\right\|_{L^{1}((0,+\infty) \times(0, T))}\right),
$$

and the estimate (149) is proved.

The improved $L^{2}\left(L^{2}\right)$ estimate (150) follows from (149), (141), and Poincaré's inequality in $H^{1}$ (see, e.g., [6]).

At this point we use Moser's iterations to obtain an $L^{\infty}\left(L^{\infty}\right)$ error estimate.

TheOREM 7. Let $O_{T}=\Omega^{+} \times(0, T)$. Then, with the notation of Theorem 6 , we have

$$
\begin{gathered}
\left\|t^{5}\left(c^{\varepsilon}-c_{1}^{e f f}(x, t ; \varepsilon)-\bar{c}_{1}^{e f f}-c_{2}^{e f f}\right)\right\|_{L^{\infty}\left(O_{T}\right)} \\
\leq C(\delta)\left(\varepsilon^{4-7 \alpha / 2-\delta} H(1-\alpha)+\varepsilon^{3 / 2-\alpha-\delta} H(\alpha-1)\right) \quad \forall \delta>0 .
\end{gathered}
$$

Remark 6. From the proof we see that $C(\delta)$ has an exponential growth when $\delta \rightarrow 0$. 
Proof. Let $M>0, \xi=c^{\varepsilon}-c_{1}^{e f f}(x, t ; \varepsilon)-\bar{c}_{1}^{e f f}-c_{2}^{e f f}$, and $\xi_{M}=\sup \left\{t^{m} \xi-M, 0\right\}$. We test the equation for $\xi$ by $\Psi^{2} \xi_{M}$ and get

$$
\begin{aligned}
& \frac{1}{2} \int_{\Omega^{+}} \Psi(x)^{2} \xi_{M}^{2}(t) d x d y+D \varepsilon^{\alpha} \int_{0}^{t} \int_{\Omega^{+}} \Psi(x)^{2}\left|\partial_{x} \xi_{M}(\tau)\right|^{2} d x d y d \tau \\
& +D \varepsilon^{\alpha-2} \int_{0}^{t} \int_{\Omega^{+}} \Psi(x)^{2}\left|\partial_{y} \xi_{M}(\tau)\right|^{2} d x d y d \tau+k \int_{0}^{t} \int_{0}^{+\infty}\left(\left.\xi_{M}\right|_{y=1}\right. \\
& \left.+M \tau^{m}\right)\left.\xi_{M}\right|_{y=1} \Psi^{2}(x) d x d \tau \leq C_{1}\left|\int_{0}^{t} \int_{\Omega^{+}} \tau^{m} \Psi(x)^{2}\right| \Phi_{3}^{\varepsilon} \mid \xi_{M} d x d y d \tau
\end{aligned}
$$

$$
+\left.\int_{0}^{t} \int_{0}^{+\infty} \tau^{m}\left|\hat{g}^{\varepsilon}\right|_{y=1} \xi_{M}\right|_{y=1} \Psi^{2}(x) d x d \tau \mid+C_{2} \varepsilon^{\alpha} \int_{0}^{t} \int_{\Omega^{+}} \tau^{2 m} \Psi(x)^{2} \xi_{M}^{2} d x d y d \tau
$$

for all $m \geq 3$, where $\tau^{m} \Phi_{3}^{\varepsilon}=-\tau^{m} \Phi_{2}^{\varepsilon}+m \tau^{m-1} \xi$. We suppose that

$k M \geq \sup _{0 \leq \tau \leq T} \tau^{m}\left\|\left.\Psi \hat{g}^{\varepsilon}(\tau)\right|_{y=1}\right\|_{L^{\infty}(0,+\infty)}=c_{0}\left(\varepsilon^{4-5 \alpha / 2} H(1-\alpha)+\varepsilon^{3(1-\alpha / 2)} H(\alpha-1)\right)$.

As in the classical derivation of the Nash-Moser estimate (see [10, pp. 181-186]) we introduce

$$
\mu(M)=\int_{0}^{T} \int_{\Omega^{+} \cap\left\{t^{m} \xi-M>0\right\}} \Psi^{2} d x d y d t .
$$

Now in exactly the same way as in [10, pp. 181-186], on a time interval which could be smaller than $[0, T]$, but assumed equal to it without losing the generality, we get

$$
\begin{gathered}
\left\|\xi_{M}\right\|_{V_{2}}^{2}=\sup _{0 \leq t \leq T} \int_{\Omega^{+}} \Psi(x)^{2} \xi_{M}^{2}(t) d x d y+D \varepsilon^{\alpha} \int_{0}^{T} \int_{\Omega^{+}} \Psi(x)^{2}\left|\partial_{x} \xi_{M}(\tau)\right|^{2} d x d y d \tau \\
+D \varepsilon^{\alpha-2} \int_{0}^{T} \int_{\Omega^{+}} \Psi(x)^{2}\left|\partial_{y} \xi_{M}(\tau)\right|^{2} d x d y d \tau \leq \beta_{0}^{2}\left\|\tau^{m} \Phi_{3}^{\varepsilon} \Psi\right\|_{L^{q}\left(O_{T}\right)}^{2} \mu(M)^{1-2 / q} \\
q>2 .
\end{gathered}
$$

Next, the estimate (157) is iterated in order to conclude that $\xi_{M}=0$. Here we modify the classical argument from [10, pp. 102-103] and adapt it to our situation.

We note that, after making appropriate extensions, one finds

$$
\|\Psi \varphi\|_{L^{4}\left(O_{T}\right)} \leq c_{0}\|\Psi \varphi\|_{L^{2}\left(O_{T}\right)}^{1 / 2}\|\Psi \varphi\|_{H^{1}\left(O_{T}\right)}^{1 / 2} \leq c_{0} \varepsilon^{-\alpha / 4}\|\varphi\|_{V_{2}}
$$

for all $\varphi \in V_{2}$, such that $\left.\varphi\right|_{x=0}=0$. As in [10, p. 102], at this point we take the sequence of levels $k_{h}=M\left(2-2^{-h}\right), h=0,1, \ldots$ Then

$$
\left(k_{h+1}-k_{h}\right) \mu^{1 / 4}\left(k_{h+1}\right) \leq\left\|\Psi \xi_{k_{h}}\right\|_{L^{4}\left(O_{T}\right)} \leq \frac{\bar{\beta} \varepsilon^{-\alpha / 4}}{k_{h+1}-k_{h}}\left\|\xi_{k_{h}}\right\|_{V_{2}}
$$

and, with $\kappa=1-2 / q>0$,

$$
\mu^{1 / 4}\left(k_{h+1}\right) \leq 2^{h} \frac{2 \bar{\beta} \beta_{0}\left\|\tau^{m} \Phi_{3}^{\varepsilon} \Psi\right\|_{L^{q}\left(O_{T}\right)} \varepsilon^{-\alpha / 4}}{M} \mu^{(1+\kappa) / 4}\left(k_{h}\right) .
$$


$\mu^{1 / 4}\left(k_{h+1}\right)$ will tend to zero for $h \rightarrow \infty$ if $\mu^{1 / 4}(M)$ satisfies

$$
\mu^{1 / 4}(M) \leq\left(\frac{2 \bar{\beta} \beta_{0}\left\|\tau^{m} \Phi_{3}^{\varepsilon} \Psi\right\|_{L^{q}\left(\Omega^{+} \times(0, T)\right)} \varepsilon^{-\alpha / 4}}{M}\right)^{-1 / \kappa} 2^{-1 / \kappa^{2}} .
$$

Equation (161) is satisfied if $M$ equals the right-hand side of estimate (153).

Our next result concerns higher order norms. It it not very satisfactory for large $\alpha$ and we state it without giving the proof, which follows from the demonstrations given above.

TheOREM 8. With the notation of Theorem 4, we have

$$
\begin{gathered}
\left\|t^{5} \partial_{x}\left(c^{\varepsilon}-c_{1}^{e f f}(x, t ; \varepsilon)-\bar{c}_{1}^{e f f}\right)\right\|_{L^{\infty}\left(0, T ; L^{2}\left(\Omega_{K}\right)\right)} \\
\leq C\left(\varepsilon^{4-15 \alpha / 4} H(1-\alpha)+\varepsilon^{(1-\alpha / 2) / 2} H(\alpha-1)\right), \\
\left\|t^{5} \partial_{t}\left(c^{\varepsilon}-c_{1}^{e f f}(x, t ; \varepsilon)-\bar{c}_{1}^{e f f}\right)\right\|_{L^{2}\left(0, T ; L^{2}\left(\Omega_{K}\right)\right)} \\
\leq C\left(\varepsilon^{4-15 \alpha / 4} H(1-\alpha)+\varepsilon^{(1-\alpha / 2) / 2} H(\alpha-1)\right) .
\end{gathered}
$$

Our final improvement concerns the error estimate in the $L^{\infty}\left(L^{2}\right)$ norm for small values of $\alpha$. As mentioned in the proof of Theorem $5, \tilde{F}_{1}^{\varepsilon}$ and $\tilde{F}_{3}^{\varepsilon}$ do not have zero means with respect to $y$, and consequently, the proof does not give the expected precision for the second-order correction. Nevertheless, when computing the term $c^{2}$ in the asymptotic expansion, there is a liberty of adding an arbitrary function $C_{2}$ of $x$ and $t$. This function can be chosen such that the appropriate means are zero and the left-hand sides of the estimates (140)-(142) are multiplied by $\varepsilon^{1-\alpha / 2}$. Unfortunately, there is a simultaneous new contribution of the form $Q P_{2}(y) \partial_{x} C_{2}$. Its norm deteriorates the estimate for $\alpha \geq 4 / 5$. Consequently, this amelioration is not of real importance and we just give the result. The proof is completely analogous to the preceding ones.

Corollary 4. Let the polynomials $P_{j}(y)$ be defined by (143)-(145) and let $C_{2}$ be given by the initial/boundary value problem

$$
\begin{aligned}
& \frac{\partial C_{2}}{\partial t}+\frac{2 Q}{3} \frac{\partial C_{2}}{\partial x}-\varepsilon^{\alpha} D \frac{\partial^{2} C_{2}}{\partial x^{2}} \\
& =-\frac{Q k}{D} \zeta(t)\left\{\partial_{x x} c \frac{Q}{D} \int_{0}^{1}\left(1-y^{2}\right) \tilde{P}_{6}(y) d y+\left(\partial_{x t} c \frac{1}{2 D}-\varepsilon^{\alpha} \partial_{x x x} c \frac{1}{2}\right) \int_{0}^{1}\left(1-y^{2}\right) P_{4}(y) d y\right. \\
& \left.+\left(\frac{Q}{45 D} \partial_{x} c-\frac{k}{6 D} c\right) \int_{0}^{1}\left(1-y^{2}\right) P_{2}(y) d y\right\} \\
& -\frac{Q^{2}}{D^{2}} \zeta(t)\left\{-\partial_{x x x} c Q \int_{0}^{1}\left(1-y^{2}\right) P_{8}(y) d y\right. \\
& \left.+\left(\partial_{x x t} c-D \varepsilon^{\alpha} \partial_{x x x x} c\right) \int_{0}^{1}\left(1-y^{2}\right) P_{6}(y) d y\right\} \text { in }(0,+\infty) \times(0, T), \\
& \partial_{x} C_{2} \in L^{2}((0,+\infty) \times(0, T)),\left.\quad C_{2}\right|_{t=0}=0,\left.\quad C_{2}\right|_{x=0}=0 .
\end{aligned}
$$

Then, with the notation of Theorem 6 and for $\alpha \in[0,4 / 5]$ we have

$$
\begin{gathered}
\left\|t^{5}\left(c^{\varepsilon}-c_{1}^{e f f}-\bar{c}_{1}^{e f f}-c_{2}^{e f f}-C_{2}\right)\right\|_{L^{\infty}\left(0, T ; L^{2}\left(\Omega_{K}\right)\right)} \leq C \varepsilon^{5-17 \alpha / 4}, \\
\left\|t^{5} \partial_{y}\left(c^{\varepsilon}--c_{1}^{e f f}-\bar{c}_{1}^{e f f}-c_{2}^{e f f}\right)\right\|_{L^{2}\left(0, T ; L^{2}\left(\Omega_{K}\right)\right)} \leq C \varepsilon^{6-19 \alpha / 4}, \\
\left\|t^{5} \partial_{x}\left(c^{\varepsilon}-c_{1}^{e f f}-\bar{c}_{1}^{e f f}-c_{2}^{e f f}-C_{2}\right)\right\|_{L^{2}\left(0, T ; L^{2}\left(\Omega_{K}\right)\right)} \leq \varepsilon^{5-19 \alpha / 4} .
\end{gathered}
$$


Acknowledgments. The authors are grateful to I. S. Pop for his assistance and they acknowledge the referees for their valuable comments.

\section{REFERENCES}

[1] R. ARIS, On the dispersion of a solute in a fluid flowing through a tube, Proc. Roy. Soc. London Ser. A, 235 (1956), pp. 67-77.

[2] L. V. Berlyand and M. V. Goncharenko, The averaging of the diffusion equation in porous medium with weak absorption, J. Soviet Math., 53 (1990), pp. 3428-3435.

[3] A. Bourgeat, M. Jurak, And A. L. Piatnitski, Averaging a transport equation with small diffusion and oscillating velocity, Math. Methods Appl. Sci., 26 (2003), pp. 95-117.

[4] R. E. CAflisch And J. Rubinstein, Lectures on the Mathematical Theory of Multiphase-Flow, Courant Institute of Mathematical Sciences, New York, 1984.

[5] C. J. van DuiJn And I. S. Pop, Crystal dissolution and precipitation in porous media: Pore scale analysis, J. Reine Angew. Math., 577 (2004), pp. 171-211.

[6] L. C. Evans, Partial Differential Equations, Grad. Stud. Math. 19, AMS, Providence, RI, 1998.

[7] E. Grenier, Boundary layers for parabolic regularization of totally characteristic quasilinear parabolic equations, J. Math. Pures Appl. (9), 76 (1997), pp. 965-990.

[8] E. Grenier And O. Gù̀s, Boundary layers for viscous perturbations of noncharacteristic quasilinear hyperbolic problems, J. Differential Equations, 143 (1998), pp. 110-146.

[9] E. Grenier and F. Rousset, Stability of one-dimensional boundary layers by using Green's functions, Comm. Pure Appl. Math., 54 (2001), pp. 1343-1385.

[10] O. A. Ladyzhenskaya, V. A. Solonnikov, and N. N. Uralceva, Linear and Quasi-Linear Equations of Parabolic Type, AMS, Providence, RI, 1968.

[11] A. J. Majda And P. R. Kramer, Simplified models for turbulent diffusion: Theory, numerical modelling, and physical phenomena, Phys. Rep., 314 (1999), pp. 237-574.

[12] R. MAURI, Dispersion, convection, and reaction in porous media, Phys. Fluids A, 3 (1991), pp. $743-756$.

[13] G. N. Mercer And A. J. Roberts, A centre manifold description of contaminant dispersion in channels with varying flow properties, SIAM J. Appl. Math., 50 (1990), pp. 1547-1565.

[14] O. A. Oleinik AND G. A. IOSIF'JAn, On the behavior at infinity of solutions of second-order elliptic equations in domains with noncompact boundary, Math. USSR Sb., 40 (1981), pp. $527-548$.

[15] M. A. Paine, R. G. Carbonell, and S. Whitaker, Dispersion in pulsed systems I. Heterogeneous reaction and reversible adsorption in capillary tubes, Chem. Eng. Sci., 38 (1983), pp. 1781-1793.

[16] A. I. Piatniski, Averaging singularly perturbed equation with rapidly oscillating coefficients in a layer, Math. USSR-Sb., 49 (1984), pp. 19-40.

[17] A. I. PiAtniski, Personal communication, 2005.

[18] J. Rubinstein And R. Mauri, Dispersion and convection in periodic porous media, SIAM J. Appl. Math., 46 (1986), pp. 1018-1023.

[19] G. I. TAYLOR, Dispersion of soluble matter in solvent flowing slowly through a tube, Proc. Roy. Soc. London Ser. A, 219 (1953), pp. 186-203. 